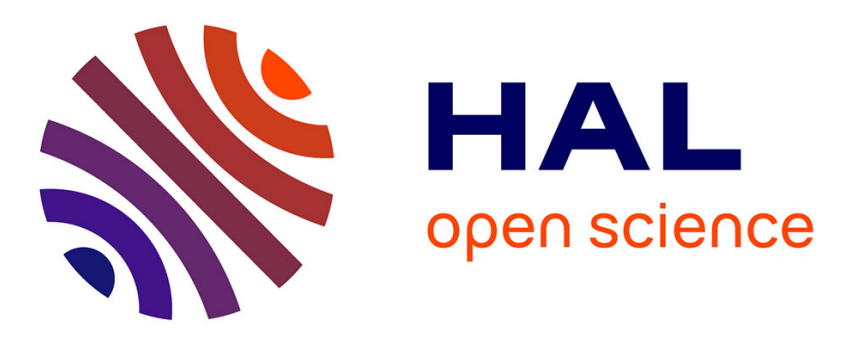

\title{
Error-Bounded and Feature Preserving Surface Remeshing with Minimal Angle Improvement
}

Kaimo Hu, Dong-Ming Yan, David Bommes, Pierre Alliez, Bedrich Benes

\section{To cite this version:}

Kaimo Hu, Dong-Ming Yan, David Bommes, Pierre Alliez, Bedrich Benes. Error-Bounded and Feature Preserving Surface Remeshing with Minimal Angle Improvement. IEEE Transactions on Visualization and Computer Graphics, 2017, 10.1109/TVCG.2016.2632720 . hal-01549584

\section{HAL Id: hal-01549584 \\ https://hal.inria.fr/hal-01549584}

Submitted on 19 Sep 2017

HAL is a multi-disciplinary open access archive for the deposit and dissemination of scientific research documents, whether they are published or not. The documents may come from teaching and research institutions in France or abroad, or from public or private research centers.
L'archive ouverte pluridisciplinaire HAL, est destinée au dépôt et à la diffusion de documents scientifiques de niveau recherche, publiés ou non, émanant des établissements d'enseignement et de recherche français ou étrangers, des laboratoires publics ou privés. 


\title{
Error-Bounded and Feature Preserving Surface Remeshing with Minimal Angle Improvement
}

\author{
Kaimo Hu, Dong-Ming Yan, David Bommes, Pierre Alliez and Bedrich Benes
}

\begin{abstract}
The typical goal of surface remeshing consists in finding a mesh that is (1) geometrically faithful to the original geometry, (2) as coarse as possible to obtain a low-complexity representation and (3) free of bad elements that would hamper the desired application. In this paper, we design an algorithm to address all three optimization goals simultaneously. The user specifies desired bounds on approximation error $\delta$, minimal interior angle $\theta$ and maximum mesh complexity $N$ (number of vertices). Since such a desired mesh might not even exist, our optimization framework treats only the approximation error bound $\delta$ as a hard constraint and the other two criteria as optimization goals. More specifically, we iteratively perform carefully prioritized local operators, whenever they do not violate the approximation error bound and improve the mesh otherwise. Our optimization framework greedily searches for the coarsest mesh with minimal interior angle above $\theta$ and approximation error bounded by $\delta$. Fast runtime is enabled by a local approximation error estimation, while implicit feature preservation is obtained by specifically designed vertex relocation operators. Experiments show that our approach delivers high-quality meshes with implicitly preserved features and better balances between geometric fidelity, mesh complexity and element quality than the state-of-the-art.
\end{abstract}

Index Terms—surface remeshing, error-bounded, feature preserving, minimal angle improvement, feature intensity.

\section{INTRODUCTION}

S Urface remeshing is a key component in many geometry processing applications such as simulation, deformation, or parametrization [1]. While many remeshing techniques are goal-specified, a common goal is to find a satisfactory balance between the following three criteria:

- The output mesh should be a good approximation of the input, making the geometric fidelity, usually measured as the approximation error, a key requirement for most applications.

- The quality of mesh elements is crucial for robust geometry processing and numerical stability of simulations, which requires fairly regular meshes in terms of both geometry and connectivity. Particularly, a lower bound on the minimal angle is vital for many simulation applications [2].

- Mesh complexity, measured as the number of mesh elements, is important for an efficient representation of complex shapes. Since mesh complexity usually conflicts with geometric fidelity and element quality, a "just enough" resolution for the required element quality and geometric fidelity should be the goal.

However, to the best of our knowledge, only a few approaches fulfill all of the above criteria. Most existing

- K. Hu is with Purdue University, 610 Purdue Mall, West Lafayette, IN 47907, US. E-mail: hukaimo02@gmail.com.

- D.-M. Yan is with the National Laboratory of Pattern Recognition, Institute of Automation, Chinese Academy of Sciences, Beijing 100190, China. E-mail: yandongming@gmail.com.

- D. Bommes is with RWTH Aachen University, Schinkelstr. 2, 52062 Aachen, Germany. E-mail: bommes@aices.rwth-aachen.de.

- P. Alliez is with Inria Sophia-Antipolis Mediterranee, 2004 route des Lucioles BP 93, 06902 Sophia-Antipolis cedex, France. E-mail: pierre.alliez@inria.fr.

- B. Benes is with Purdue University, 610 Purdue Mall, West Lafayette, IN 47907, US. E-mail: bbenes@purdue.edu. methods that generate meshes with high element quality often require high mesh complexity or introduce high approximation error [3], [4]. The error-driven methods, while preserving the results in controllable geometric fidelity and low mesh complexity, do not deal with the element quality [5], [6]. In addition, many approaches require the sharp features to be specified or detected in advance [7]-[9], which is usually difficult and error-prone.

We propose an approach that controls both the approximation error and element quality simultaneously. Our approach only requires the user to specify the error-bound threshold $\delta$, the desired minimal angle $\theta$ and an upper bound on the mesh complexity $N$, measured as the number of vertices. In an initial phase our algorithm coarsens the mesh as much as possible while respecting the error-bound $\delta$. It then iteratively improves the minimal angle of the mesh until the desired bound for $\theta$ or $N$ is met. Since all atomic operations respect the error-bound $\delta$, the result is guaranteed to satisfy geometric fidelity. In contrast, low mesh complexity and high quality of mesh elements are optimization goals, which depending on the model and the desired bounds might or might not be met. However, experiments show that given a reasonable vertex budget $N$ and an angle bound $\theta \leq 35^{\circ}$ our algorithm is usually able to reach all three goals simultaneously. Moreover, our method preserves geometric features like sharp creases and ridges without explicitly specifying or detecting them.

Our method is inspired by the remeshing methods that proceed by applying a series of local operators such as edge split, edge collapse, edge flip, and vertex relocate [10], [11]. Contrary to the existing methods that iteratively apply these operations sequentially and globally, our core algorithm employs a dynamic priority queue to maintain all angles which are smaller than the user specified threshold, and 


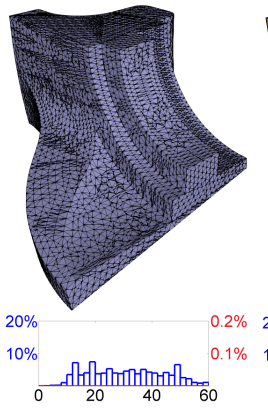

(a) Input model.

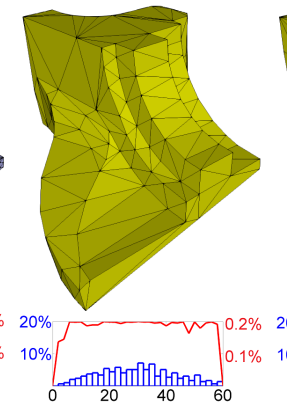

(b) $\theta=0^{\circ}$.

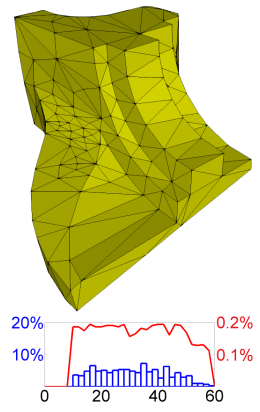

(c) $\theta=10^{\circ}$.

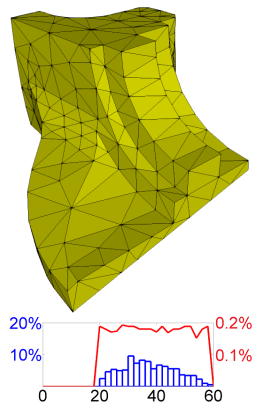

(d) $\theta=20^{\circ}$.

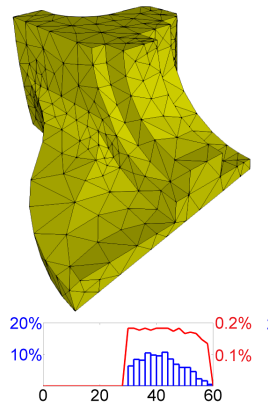

(e) $\theta=30^{\circ}$.

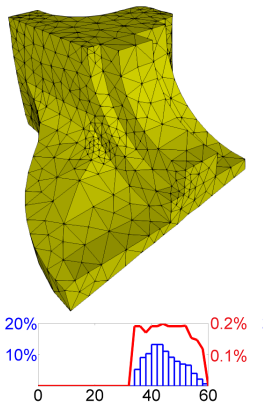

(f) $\theta=35^{\circ}$.

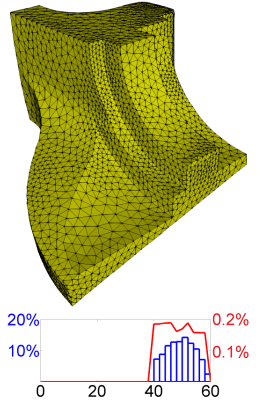

(g) $\theta=40^{\circ}$.

Fig. 1. Examples of surface meshes generated with our approach. The input model (a) has 7.2k vertices. From (b) to (g) are the results with different minimal angle threshold $\theta$. The angle distributions and their corresponding approximation error are shown below each result, in blue bars and red curves respectively. The error-bound threshold $\delta$ is set to $0.2 \%$ of the diagonal length of the input's bounding box (\%bb).

then greedily applies local operators whenever an improvement of the mesh is possible. After the initial coarsening our method only improves the mesh in local regions where the element quality is poor, and thus modifies the model as little as necessary with respect to the minimal angle threshold $\theta$ and the error-bound constraint $\delta$.

Since we apply local operators directly on the mesh, without using any surface parameterizations or density functions, our algorithm is naturally suitable for high genus, irregular, and multi-component inputs, as well as meshes with very high/low resolutions. In summary, we claim the following contributions:

- A surface remeshing algorithm with minimal angle improvement based on applying local operators, which bounds the "worst element quality" (Sec. 3).

- A reliable and efficient local error update scheme based on approximated symmetric Hausdorff distance, which bounds the geometric fidelity (Sec. 4).

- Two feature intensity functions designed for vertex position relocation, which enable implicit feature preservation and support geometric fidelity (Sec. 5).

\section{Related Work}

The variety of applications leads to a large number of different remeshing techniques. We restrict the discussion to the most relevant aspects of our algorithm, i.e. high-quality remeshing, error-driven remeshing and feature-preserving remeshing. For a more complete discussion we refer the reader to the survey [12].

High quality remeshing is typically based on sampling and Centroidal Voronoi Tessellation (CVT) optimization [13]. Early approaches apply 2D CVT in a parametric domain [3], [7], [14]-[17]. Instead of CVT optimization, Vorsatz et al. [18] utilize a partial system approach in the parametric domain. In general, parametrization-based methods suffer from the additional distortion of the map and the need to stitch parameterized charts for high genus surfaces. Valette et al. [4] perform a discrete version of CVT directly on the input surface. However, the resulting mesh quality can be poor due to the inexact computation. Yan et al. [8], [19], [20] avoid the parameterization by computing the 3D CVT restricted to the surface. Additionally, they proposed blue-noise remeshing techniques using adaptive maximal
Poisson-disk sampling [9], [21], farthest point optimization [22], and push-pull operations [23], which improve the element quality as well as introducing blue-noise properties. However, these approaches still suffer from common limitations, e.g., geometric fidelity and the minimal angle cannot be explicitly bounded. Moreover, sharp features must be specified in advance.

Another way to avoid the stitching problem is to operate directly on the surface mesh [24], [25]. An efficient isotropic approach proposed by Botsch and Kobbelt [11] takes an edge length as input and repeatedly splits long edges, collapses short edges, equalizes vertex valences and relocates vertex positions until all edges are approximately of the specified target edge length. To extend this work to an adaptive version, Dunyach et al. [26] replace the constant target edge length with an adaptive sizing field that is sensitive to local curvatures. Since this kind of methods requires neither surface parameterization nor density functions, they are easy to implement, robust for high genus inputs, and efficient for real-time applications. Our method falls into this category. However, we enrich the local operators and apply them in a more selective manner in order to obtain guarantees on the geometric fidelity as well as higherquality results and implicit feature preservation.

Error-driven remeshing amounts to generating a mesh that optimizes the tradeoff between geometric fidelity and mesh complexity. Cohen-Steiner et al. [5] propose an error-driven clustering method to coarsen the input mesh. They formulate the approximation problem as a variational geometric partitioning problem, and optimize a set of planes iteratively using Lloyd's iteration [27] to minimize a predefined approximation error.

The mesh simplification techniques are similar to errordriven remeshing in some way. Garland and Heckbert [28] use iterative contractions of vertex pairs to simplify models and maintain surface approximation error based on quadric error metrics. Borouchaki and Frey [29] define a fidelity metric named Hausdorff envelope, and simplify and optimize the reference mesh that stays inside the tolerance volume. While they consider the geometric fidelity and element quality simultaneously, nothing is done to improve the worst element quality. Our method guarantees the worst element quality by improving the minimal angle and keeping the mesh complexity as low as possible, with respect to a given error-bound. Based on the concept of tolerance vol- 
ume, Mandad et al. [6] propose an isotopic approximation method. Their algorithm generates a surface triangle mesh guaranteed to be within a given tolerance volume. However, they generate the mesh from scratch, and mainly focuse on mesh complexity rather than element quality. Instead, we strive for good balances between mesh complexity and element quality with a given input.

Feature preservation is crucial in remeshing. However, automatically identifying sharp features on a surface mesh is a difficult problem that depends both on the local shape and on the global context and semantic information of the model. This makes feature detection a strongly ill-posed problem. A wide range of approaches address this problem [30]-[32]. However, none of them works reliably for all kinds of meshes. Most remeshing algorithms avoid this problem by assuming that the features have been specified in advance [8], [9], [19], [21], [22], [33]. Some remeshing techniques try to preserve features implicitly [34], [35]. Vorsatz et al. [36] first apply a relaxation in the parameter domain, and then snap the vertices to feature edges and corners. Since they separate remeshing and feature-snapping, the resulting mesh quality near sharp features might be poor. Valette [4] alleviates this issue by embedding the Quadric Error Metric approximation (QEM) criterion inside the CVT optimization. However, the performance of their clusterbased method is highly dependent on the quality of the input, and the sharp features might not be well preserved when users specify a small vertex budget. Jakob et al. [37] propose a general framework for isotropic triangular/quaddominant remeshing using a unified local smoothing operator, in which the edges naturally align to sharp features. However, little attention is paid on the approximation error and element quality. We address this problem by optimizing the element quality explicitly in combination with implicit feature preservation based on the new defined feature intensity functions.

\section{ALgorithm OVERVIEW}

Given a 2-manifold triangular mesh $M_{I}$, the goal of our algorithm consists in finding an improved surface mesh $M_{R}$ with approximation error below $\delta$, minimal interior angle above $\theta$ and mesh complexity below $N$. The main idea is to transform the mesh by a series of discrete local operators as illustrated below:

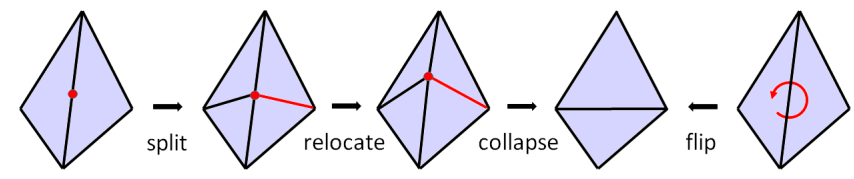

Fig. 2. Common local operators [1].

In our algorithm, only edge collapse, edge split and vertex relocation operators are employed. Edge flips are implicitly performed as a combination of an edge split followed by an edge collapse (cf. Fig. 2). This convention not only lowers the combinatorial complexity of all operators but is also advantageous for our implicit feature preservation since edge flips tend to destroy sharp creases and would require additional nontrivial checks.
The remeshing algorithm is designed to perform local operators in a conservative manner. More specifically, a local operator is only executed if it respects the approximation error bound $\delta$, does not introduce new interior angles below current minimal angle $\theta_{\min }$ and maintains the 2manifoldness of the mesh. This behavior can be interpreted as a conservative greedy algorithm, which in each step identifies the most promising operation that improves the result (either coarsens or improves angles), while never leaving the feasible set of meshes with approximation error below $\delta$. Note that since pure topological edge collapses and edge splits improve the mesh quality very rarely, these operations are always combined with vertex position optimization.

The most crucial design choices of the algorithm are the scheduling of different operators and the efficient modeling and handling of approximation error queries (cf. Sec.4). The algorithm passes through three different stages:

The initial phase concentrates on coarsening and runs a mesh simplification, which solely performs edge collapses.

The second phase then tries to lift the minimal interior angle above the user-provided bound $\theta$. For this task we devised three different processes, which are tried subsequently, as illustrated in Fig. 3. Since an edge collapse reduces the complexity, it would be the best way to improve the minimal angle. If edge collapse is not possible, we try to improve the minimal angle by relocating one of the triangle's vertices. If vertex relocation also fails, edge splits are considered as the last option as they increase the mesh complexity. While edge splits do not directly improve the minimal angle, they are crucial to enrich the local mesh connectivity in order to enable improvements in subsequent steps. We apply an approach similar to the longest-side propagation path [38], described in more detail in Sec. 3.2.

The third stage of our algorithm freezes the mesh connectivity and concentrates on global vertex relocation. This stage is designed to improve the average quality of the mesh elements, while not violating the user-specified bounds. The pseudocode of our remeshing algorithm is shown in Alg. 1.

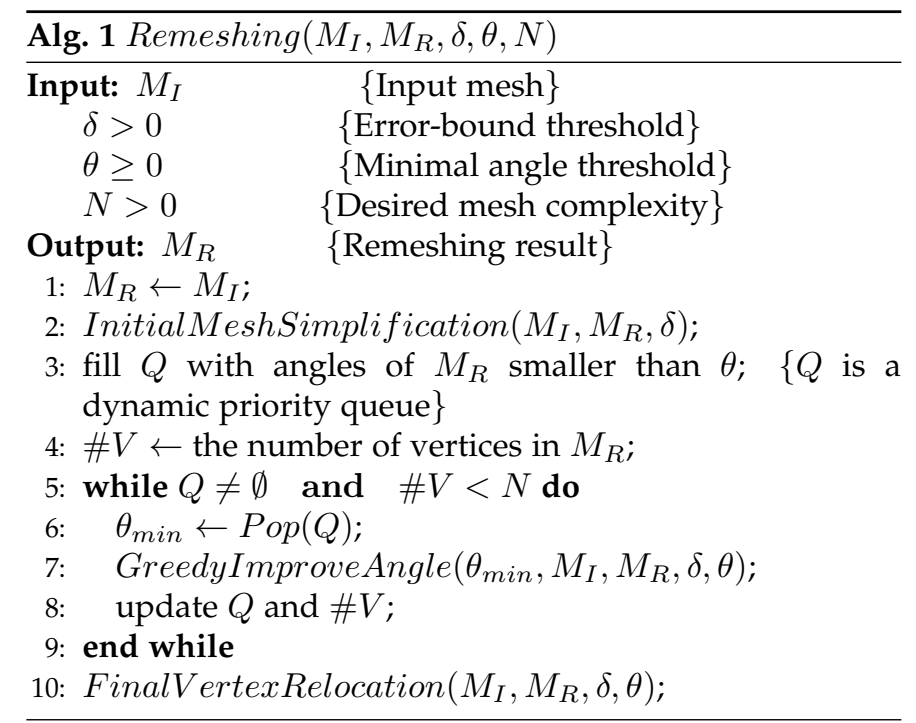

In the following we provide more details on the three phases of initial mesh simplification, greedy angle improvement and vertex relocation. 


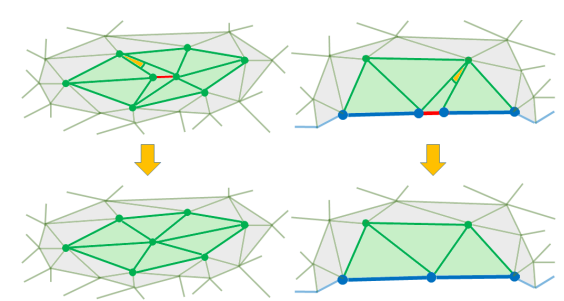

(a) Edge collapse.

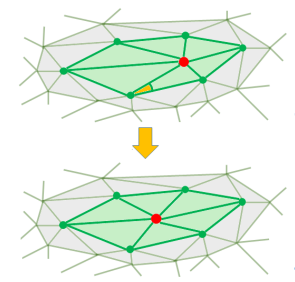

(b) Vertex relocation.

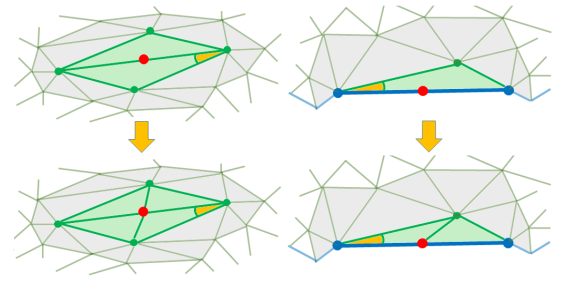

(c) Edge split.

Fig. 3. Local operators. The local patches before/after applying the operators are shown in the first/second rows, respectively. The inner patch (green) contains the facets that will be directly affected by the local operators, and the outer patch (gray) includes the facets who share vertices with facets of the inner patch. In each sub figure, the left shows the inner case while the right shows the boundary case, in which the boundary edges and vertices are depicted in blue. We depict the current minimal angle $\theta_{\min }$ in yellow.

\subsection{Initial Mesh Simplification}

The input mesh is often densely sampled such that the mesh complexity can be significantly reduced without violating the approximation error bound. While such a mesh simplification could potentially also be done on the fly, a specialized pre-process turns out to be more efficient. The goal of this step consists in finding a mesh, which is significantly coarser and offers a good starting point for the second phase of element quality improvement. Simplification is achieved through iteratively collapsing edges and relocating related vertices as long as the approximation error does not exceed $\delta$. Short edges, or those opposite to small angles are likely to lower the mesh quality and are consequently collapsed first. This is achieved through using a priority queue sorted by increasing values of the following function: $l(h) \cdot\left(\theta_{1}(h)+\theta_{2}(h)\right) / 2$, where $l(h)$ denotes the length of halfedge $h$ and $\theta_{i}(h)$ denote the two angles opposite to $h$ and $h$ 's opposite halfedge. The pseudocode of the corresponding function is in Alg. 2.

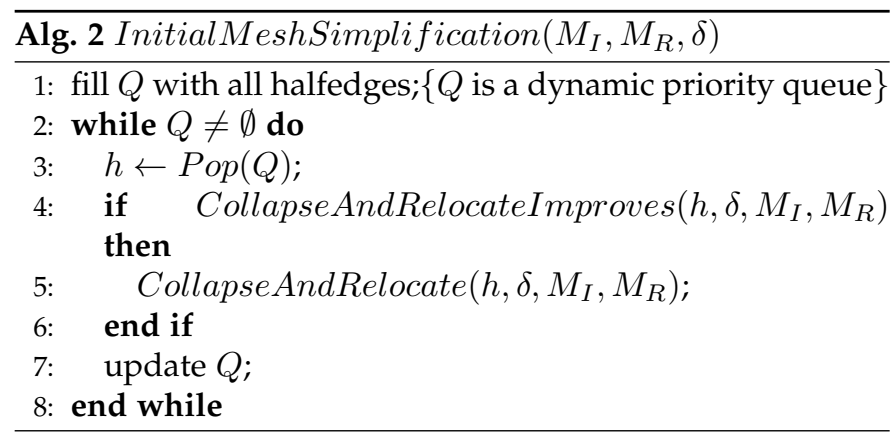

\subsection{Greedy Improvement of Angles}

The second phase is designed to improve the mesh quality by iteratively increasing the smallest angle in the mesh, until the desired angle bound $\theta$ is satisfied or the complexity limit $N$ is reached. Our approach repeats the following process: We simulate a potential operation, test whether the resulting mesh improves and only in this case perform the candidate operation. The mesh improvement test additionally contains several important validity checks.

Mesh Improvement Test: we simulate each potential operation and measure if the following constraints are satisfied:

- Topology. For edge collapses topology changes are prevented by checking the link condition [39].
- Geometry. The operator should not create fold-overs by flipping the orientation facets (cf. Fig. 4).

- Fidelity. The approximation error between $M_{R}$ and $M_{I}$ should remain below $\delta$ (cf. Fig. 6).

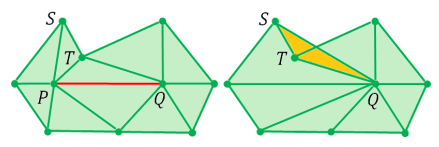

(a) Edge collapse.

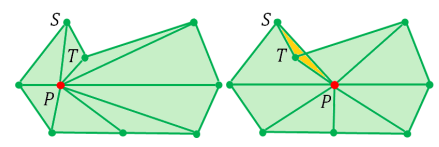

(b) Vertex relocate.
Fig. 4. Creation of fold-overs in 2D. (a) Edge $P Q$ will be collapsed into Q. $\triangle S T Q$ creates fold-overs if $P$ and $Q$ lie on the two sides of line $S T$; (b) Vertex $P$ will be relocated. $\triangle S T P$ creates fold-overs if $P$ before and $P$ after relocation are located on the two sides of line $S T$. In 3D, we say that a triangle creates fold-overs if its normals before and after applying a local operator have opposite orientations.

In order to improve the minimal angle, we greedily apply operators that pass the mesh improvement test. The operators are tested in the following order: edge collapses, vertex relocations and edge splits. The pseudocode of the greedy improvement is shown in Alg. 3.

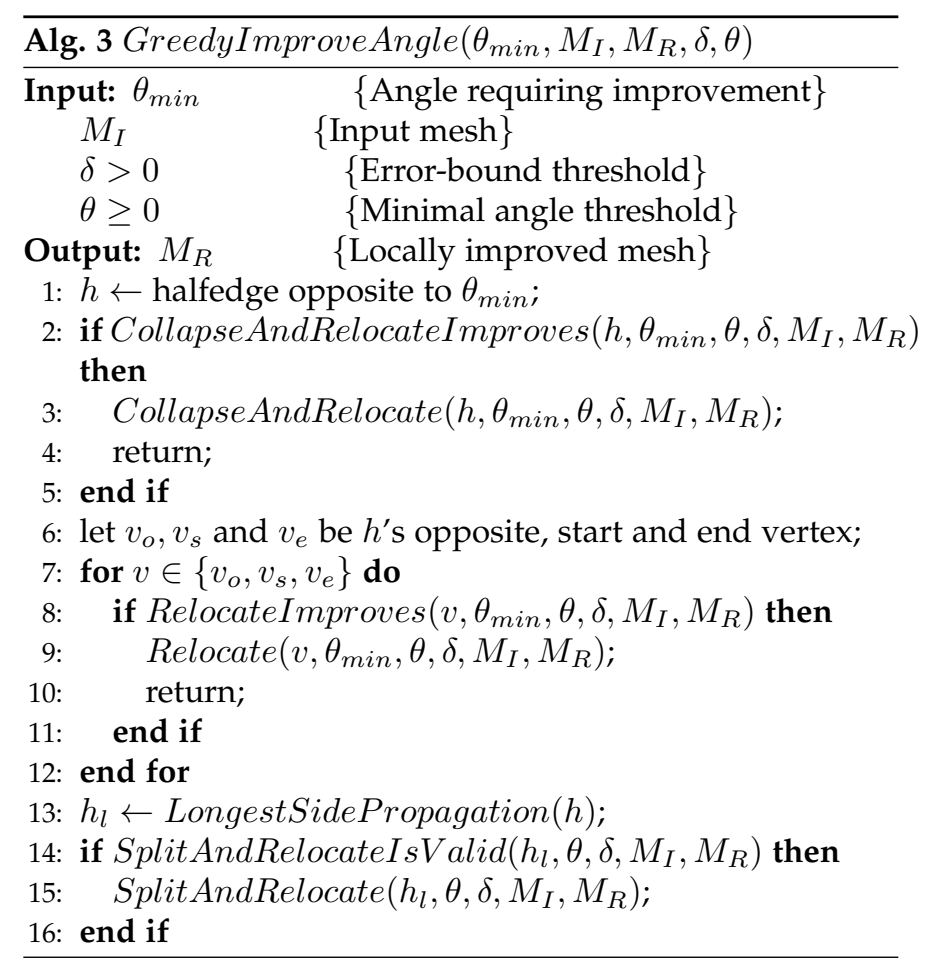


Longest-Side Propagation Path: If neither edge collapse nor vertex relocation is possible, we search the longest edge $h_{l}$ in the neighborhood [38] and then split it. Starting from an edge $e$, we iteratively move on to the longest edge of the neighboring two triangles until no further enlargement is possible or we hit the boundary. Though the edge split operator does not increase $\theta_{\min }$ directly, it modifies the local connections such that $\theta_{\min }$ can be improved in later iterations (cf. Fig. 3(c)). While there might exist other ways for local connectivity modification, experiments show that this strategy works well in our algorithm.

\subsection{Final vertex relocation}

To achieve a better overall element quality, in the third phase we perform a series of vertex relocations until the angles can no longer be significantly improved. In contrast to the second phase which only focuses on specific regions where angle improvement is required, the third stage optimizes all vertex locations. We maintain the relocation candidate set in a queue, which is initialized with all vertices. Whenever a vertex is relocated, all its neighbors are added to the queue, since a change in the neighborhood might enable further improvements. The pseudocode of the corresponding function is shown in Alg. 4.

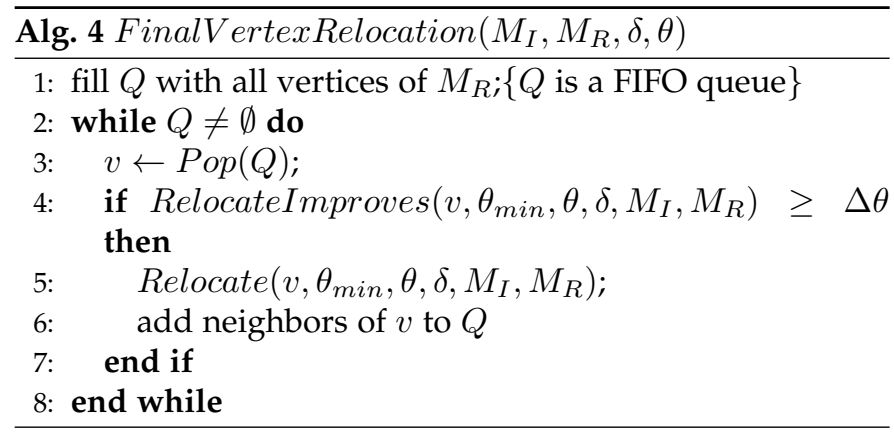

Optimal vertex positions would be found if no vertex could be relocated to a better position anymore. However, we empirically restrict the angle improvement $\Delta \theta=$ $0.1^{\circ}$ (cf. Sec. 6.4), since afterward there are usually no significant improvements anymore.

\section{Error Metric}

\subsection{Hausdorff Distance}

We use the Hausdorff distance to measure the approximation error between $M_{R}$ and $M_{I}$. Let $d(p, q)$ denote the Euclidean distance between two points $p$ and $q$ in 3D space. The distance of a point $p$ to a surface $M$ is defined as the shortest distance between $\mathrm{p}$ and any point of $M$

$$
d(p, M)=\min _{q \in M} d(p, q) .
$$

The one-sided Hausdorff distance from a source surface $M$ to a target surface $T$ is defined as the maximum of all such point to surface distances :

$$
d_{h}(M, T)=\max _{p \in M} d(p, T) .
$$

The one-sided Hausdorff distance is in general not symmetric, i.e. $d_{h}(M, T) \neq d_{h}(T, M)$. It is easily possible to construct counter-intuitive cases where $d_{h}(M, T)=0$ but $d_{h}(T, M)$ is arbitrarily large ${ }^{1}$.

The two-sided Hausdorff distance [40] between $M$ and $T$ resolves this issue by symmetrization

$$
d_{H}(M, T)=\max \left\{d_{h}(M, T), d_{h}(T, M)\right\} .
$$

\subsection{Approximating $d_{H}$ with Stratified Sampling}

The exact evaluation of the two-sided Hausdorff distance is computationally very expensive [41]. However, by careful surface sampling in combination with local updates of shortest point-to-surface links it is possible to obtain an efficient yet sufficiently accurate approximation, as discussed next.

Assume that $M$ is sampled by a point set $S_{M} \subset M$. Then the one-sided Hausdorff distance can be approximated by

$$
d_{h}(M, T) \approx \max _{a \in S_{M}} d(a, T) .
$$

By additionally sampling $T$ we obtain an approximation of the two-sided Hausdorff distance

$$
d_{H}(M, T) \approx \max \left(\max _{a \in S_{M}} d(a, T), \max _{b \in S_{T}} d(b, M)\right),
$$

with $S_{T}$ being a set of point samples on $T$. Note that our approximation still measures the exact distance from sample points to the complete surface, which provides significantly higher accuracy than a point cloud distance $d_{H}\left(S_{M}, S_{T}\right)$. Moreover, it ensures that we strictly underestimate the real distance. Following the triangle inequality, the approximation error of our sampled Hausdorff distance is bounded by $\max \left\{d_{h}\left(M, S_{M}\right), d_{h}\left(T, S_{T}\right)\right\}$, i.e. the maximum gap between sample points. Consequently, in order to guarantee a good approximation, we target a uniform sampling of the surfaces. However, for piecewise linear triangle meshes the maximal distance often occurs at creases or corners, i.e. at mesh edges or vertices. This two observations motivate our stratified sampling approach, which is uniform on faces but additionally adds samples on edges and vertices, as a kind of greedy importance sampling.

Sampling the facets. Instead of uniformly sampling the complete surface, we empirically found that better results can be obtained by uniformly sampling per triangle. In this way we obtain an automatic adaptivity, since more samples are available in structurally complex and highly curved areas, where also more triangles are necessary to describe such shape. Since our meshes are often highly non-uniform, we add a slight local smoothing of the sampling density by

$$
n\left(f_{i}\right)=n_{f} \cdot \frac{1+\left|\mathcal{N}_{f_{i}}\right|}{1+\sum_{f_{j} \in \mathcal{N}_{f_{i}}} \frac{\mathcal{A}_{j}}{\mathcal{A}_{i}}},
$$

where $n_{f}$ is the average number of samples per facet specified by the user, $\mathcal{N}_{f_{i}}$ are the neighbor facets that share vertices with $f_{i}$, and $\mathcal{A}_{i}$ is the area of facet $f_{i}$. Choosing a large

1. Choose $T$ as a sphere of radius $r$ and $M$ as a hemisphere subset. Then with $r \rightarrow \infty$ also $d_{h}(T, M) \rightarrow \infty$. 
$n_{f}$ offers a tighter approximation of the Hausdorff distance, however, resulting in high computational complexity. In our experiments, we found that $n_{f}=10$ gives a good tradeoff between efficiency and effectiveness (cf. Fig. 12).

To distribute the samples evenly on a triangle $f_{i}$, we first generate $n\left(f_{i}\right)$ samples randomly, and then perform a Lloyd relaxation process onto a bounded Voronoi Diagram (BVD) [42] (Fig. 5(b)). Usually, five iterations are sufficient to generate quasi-uniformly distributed samples on facets.

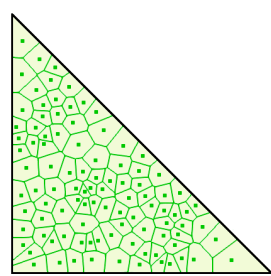

(a)

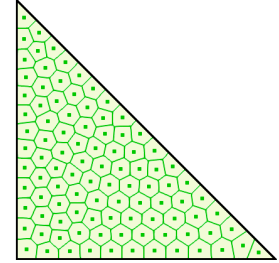

(b)

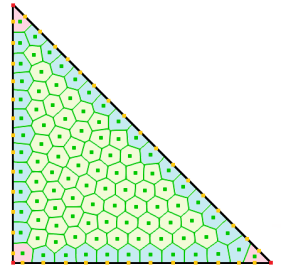

(c)
Fig. 5. Stratified sampling process. (a) Initial facet samples; (b) Optimized facet samples with BVD; (c) Edge samples. The facet, edge, and vertex samples are rendered in green, yellow and red, respectively.

Sampling the edges. By counting the number of incident Voronoi cells to an edge we first estimate the local sampling density. The resulting number of samples is then evenly distributed along the edge (Fig 5(c)).

Sampling the vertices. The position of a vertex is simply regarded as its own vertex sample.

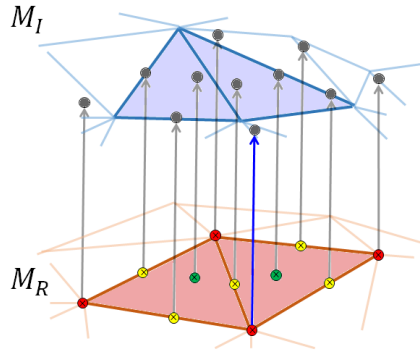
(a) Stratified samples on $M_{R}$ and their shortest links to $M_{I}$

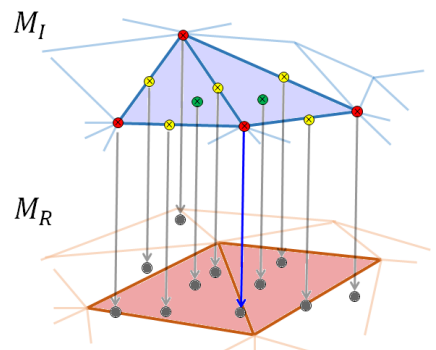

(b) Stratified samples on $M_{I}$ and their shortest links to $M_{R}$.
Fig. 6. Hausdorff distance approximation with stratified samples. Samples on vertices, edges, and faces are rendered in red, yellow, and green respectively. We use the circles centered with crosses to indicate the samples on one mesh, and the circles centered with dots to indicate their closest points on the other mesh. The approximated one-side Hausdorff distances are shown as the blue links, and the overall approximated Hausdorff distance is simply the length of the longer one.

By setting $S_{I}$ and $S_{R}$ as the stratified samples on $M_{I}$ and $M_{R}$, the Hausdorff distance between $M_{I}$ and $M_{R}$ is approximated using Eq. (5), as illustrated by Fig. 6.

\subsection{Local Update Scheme}

Each operator of our remeshing algorithm only changes a local area $L \subset M_{R}$ of the target mesh $M_{R}$ (cf. Fig. 3 green area). Hence, it is possible to rapidly compute the Hausdorff distance for the updated mesh. In general both directions of the Hausdorff distance change and require an update.
Updating $d_{h}\left(M_{R}, M_{I}\right)$ : First of all notice that the one-sided Hausdorff distance can be decomposed w.r.t. to a modified local area $L \subset M_{R}$ into

$$
d_{h}\left(M_{R}, M_{I}\right)=\max \left\{d_{h}\left(L, M_{I}\right), d_{h}\left(\bar{L}, M_{I}\right)\right\}
$$

with $M_{R}=L \cup \bar{L}$ being a partition of $M_{R}$. Since $\bar{L}$ is unchanged, checking $d_{h}\left(L, M_{I}\right) \leq \delta$ is sufficient to verify that the modification does not violate the approximation error bound $\delta$. Thus, we first re-sample the modified local area $L$ with stratified samples $S_{L}$, and then efficiently evaluate the approximated Hausdorff distance $d_{h}\left(S_{L}, M_{I}\right)$ with a pre-computed axis aligned bounding box tree [43] for the static mesh $M_{I}$.

Updating $d_{\mathbf{h}}\left(\mathbf{M}_{\mathbf{I}}, \mathbf{M}_{\mathbf{R}}\right)$ : Checking the opposite direction is more difficult for two reasons. Firstly, since $M_{R}$ changes, we cannot simply pre-compute a static search tree. Secondly, decomposing the Hausdorff distance in the second argument is more intricate. In addition to $M_{R}, M_{I}$ must also be decomposed correctly. More specifically, we have

$$
d_{h}\left(M_{I}, M_{R}\right) \approx d_{h}\left(S_{I}, M_{R}\right)=\max \left\{d_{h}\left(S_{I}^{L}, L\right), d_{h}\left(S_{I}^{\bar{L}}, \bar{L}\right)\right\}
$$

where $S_{I}=S_{I}^{L} \cup S_{I}^{\bar{L}}$ is a partitioning of samples on $M_{I}$ into those closer to $L$ and $\bar{L}$ (the rest of $M_{R}$ ) respectively.

Identifying the correct partitioning of samples $S_{I}$ would in general require global tests and is thus time consuming. Therefore, in order to enable a rapid update we only approximate this decomposition by tracking the history of samples in $S_{I}$. The key idea is to store for each sample $s_{j} \in S_{I}$ a link to its closest triangle $t_{j} \in M_{R}$ (Fig. 6). After a local modification of $M_{R}$ these links will typically change in the vicinity of the modified area $L$. Thus, in order to avoid global checks, we only update the links of samples connecting to a region $L^{+}$, which enlarges $L$ by an additional ring of neighboring triangles (cf. Fig. 3 gray area). These new links can be efficiently found by constructing and querying an the axis aligned bounding box tree of the local area $L^{+}$. Note that the resulting approximated Hausdorff distance is a strict overestimator because shorter links to $\overline{L^{+}}$ might exist that are not investigated by our approximation. A positive side effect of our localized update scheme is that links cannot jump between geodesically far away regions, which could cause topologically inconsistent links.

\section{Implicit Feature Preservation}

A clean representation of geometric features as for instance sharp creases is important in many applications ranging from visualization to simulation. In a polygonal mesh, we distinguish between vertex and edge features. Vertex features include tips, darts, cusps and corners, while edge features are either creases or boundaries. Typical examples are depicted in Fig. 7.

Instead of requiring an explicit tagging of features as most remeshing approaches, e.g. [8], [9], [19], [22], [33], our goal is to implicitly preserve features. This not only releases users from the time-consuming burden of manual tagging but moreover often enables the recovery of features that were lost, e.g. through inappropriate sampling by a 3D laser 


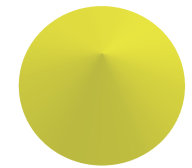

(a) tip.

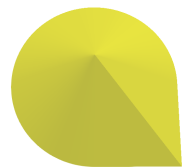

(b) dart.

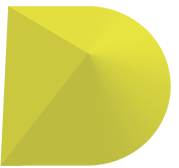

(c) cusp.

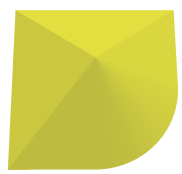

(d) corner.
Fig. 7. Vertex features: (a) Tips, (b) Darts, (c) Cusps and (d) Corners are feature vertices who are adjacent to zero, one, two and three sharp creases respectively.

scanner.

In principle feature preservation could be simply a byproduct of approximation error minimization since incorrectly meshing features usually induces a large approximation error. Nevertheless, there are several reasons why special care is still required. First of all, the minimization of the approximation error is a non-convex problem such that bad initializations might lead to low-quality local minima, not well representing features. Moreover, anticipating feature locations and placing vertices accordingly will speed up the overall method. In our method special feature handling is done whenever vertices are either newly placed or relocated, i.e. during (i) edge collapse, (ii) edge split and (iii) vertex relocation. Since robust and automatic feature detection is a difficult yet unsolved problem, we rely on a softer identification of features by means of a feature intensity function defined at vertices of the mesh.

\subsection{Feature Intensity}

Feature vertices can be characterized by large Gaussian curvature $\mathcal{K}(v)$, which in the discrete setting is identical to the angle defect, i.e.

$$
\mathcal{K}(v)= \begin{cases}\pi-\theta_{\text {sum }}(v) & \mathrm{v} \text { is on a boundary, } \\ 2 \pi-\theta_{\text {sum }}(v) & \text { otherwise }\end{cases}
$$

where $\theta_{\text {sum }}(v)$ is the sum of interior angles adjacent to $v$.

Feature edges are characterized by large dihedral angles. Accordingly, for a vertex we define the feature edge intensity $\mathcal{E}(v)$ to be the maximal unsigned dihedral angle of an edge adjacent to $v$

$$
\mathcal{E}(v)=\max _{e \in \mathcal{N}_{e}(v)}|\mathcal{D}(e)|
$$

where $\mathcal{N}_{e}(v)$ are the edges adjacent to $v$ and $\mathcal{D}(e)$ is the dihedral angle at $e$.

Finally, the feature intensity $\mathcal{F}(v)$ is defined as the combination

$$
\mathcal{F}(v)=(\tau(|\mathcal{K}(v)|)+1) \cdot(\tau(\mathcal{E}(v))+1)-1
$$

with the transfer function $\tau(x)=\min \{\pi, 2 \cdot x\}$, which rescales values by a factor of 2 and clamps them at $\pi$. Thus, the feature intensity is a value between 0 and $\left((\pi+1)^{2}-1\right)$ and corresponds to a logical or whenever one of the individual intensities vanishes. Fig. 8 shows an examples of the three fields $|\mathcal{K}|, \mathcal{E}$ and $\mathcal{F}$.

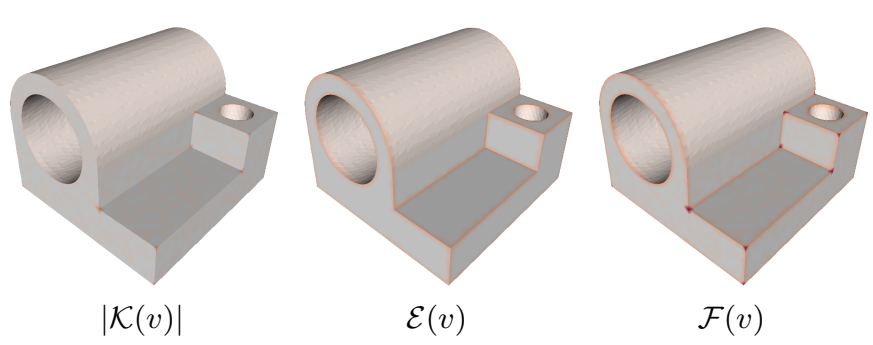

Fig. 8. From left to right Gaussian curvature $|\mathcal{K}|$, feature edge intensity $\mathcal{E}$ and combined feature intensity $\mathcal{F}$, with higher intensities in red.

\subsection{Feature-Sensitive Vertex Relocation}

In our remeshing algorithm each local operation is combined with a subsequent relocation of the modified vertex in order to minimize the approximation error. This relocation of a single vertex is done in two stages. First a feature-sensitive initialization, specifically adapted to the local operation, and then a nonlinear minimization of the two-sided Hausdorff distance. A careful initialization of vertex positions is important to avoid poor local minima of the non-convex Hausdorff energy and additionally increases performance.

Edge Collapse Initialization: During an edge collapse of edge $e_{i j}$ the two vertices $v_{i}$ and $v_{j}$ merge into a new vertex $v_{m}$ and a new position for $v_{m}$ must be specified. One feature-sensitive standard technique uses error quadrics [4], [28]. However, since we anyway perform a more accurate approximation error-driven relocation subsequently, a simpler and faster position initialization based on our feature intensity is sufficient. We distinguish two cases. If the edge is incident to a single strong feature we want that $v_{m}$ snaps onto this feature, meaning that the default behavior is to snap to the vertex $v_{k}$ with higher feature intensity, i.e. $v_{k}=\arg \max _{v \in\left\{v_{i}, v_{j}\right\}} \mathcal{F}(v)$. An unclear situation arises, when both feature intensities are similar, i.e. $\mathcal{F}\left(v_{i}\right) \approx \mathcal{F}\left(v_{j}\right)$. This happens either in regions without features or for edges along a crease, where it is reasonable to initialize $v_{m}$ to the edge midpoint. We decide for the midpoint initialization based on a parameter $\omega$, whenever

$$
\left|\mathcal{F}\left(v_{i}\right)-\mathcal{F}\left(v_{j}\right)\right|<\omega \cdot \max \left(\mathcal{F}\left(v_{i}\right), \mathcal{F}\left(v_{j}\right)\right)
$$

with $\omega=0.15$ in all our examples.

Edge Split Initialization: Since an edge split does not change the geometry we simply always initialize the new point as the edge midpoint. Further improvement is achieved through a nonlinear minimization of the twosided Hausdorff distance, as discussed next.

Vertex Relocation Initialization: Without topological mesh modifications (edge collapse or split), a vertex relocation is initialized in the following way. The goal is to anticipate a good relocation position for $v$ while preserving feature corners and creases. Therefore, we first classify the vertex $v$ as either being (i) a feature vertex, (ii) a crease vertex or (iii) a smooth vertex. If $v$ is a feature vertex it simply remains at its position. If it is on a crease, we relocate $v$ to the CVT barycenter of its two neighboring crease vertices. 
And only if it is a smooth vertex we relocate it to the CVT barycenter of all one-ring neighbors. The classification is done by counting how many neighbors $v_{i}$ are of similar or higher importance as $v$, i.e. if $\mathcal{F}\left(v_{i}\right) \geq \zeta \cdot \mathcal{F}(v)$ for a tolerance parameter $\zeta \in(0,1)$. In this way $v$ is classified as (feature vertex/crease vertex/smooth vertex), depending on whether (none/two/all) of its neighbors are of similar feature intensity. Unclear cases where $k$ out of $n$ neighbors are of similar importance are classified towards the closer possibility, meaning as a crease vertex if $k$ is closer to 2 or as a smooth vertex if $k$ is closer to $n$. Wrong classifications where two crease vertices are connected by a noncrease edge can be avoided by only counting important neighbors that are connected by an important edge with $\mid D\left(e_{v, v_{i}} \mid+1 \geq \zeta \cdot(\mathcal{E}(v)+1)\right.$, as illustrated in Fig. 9 .

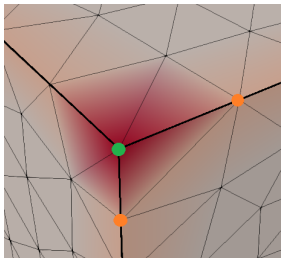

(a)

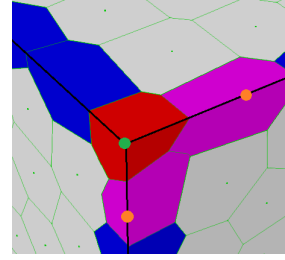

(b)

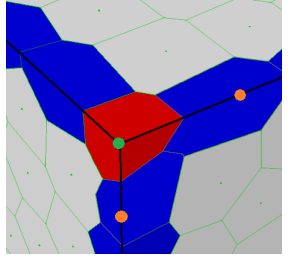

(c)
Fig. 9. Visualization of $\mathcal{F}(v)$ in (a). The color of $v$ 's Voronoi cell represents the number of important neighbors, with red $=0$, blue $=2$, purple $=3$ and gray=degree $(v)$ is shown in (b) and (c). A wrong classification based on solely feature intensity in (b) is corrected by additionally checking importance of edges in (c).

The parameter $\zeta$ controls the feature classification. For higher values of $\zeta$ more vertices are implicitly treated as features and thus prevented from free movement. Fig. 10 shows an example classification for $\zeta$ varying between 0.3 and 0.7 . In our experiments the default is $\zeta=0.5$

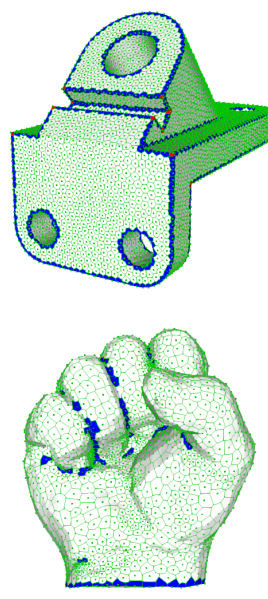

$\zeta=0.3$.
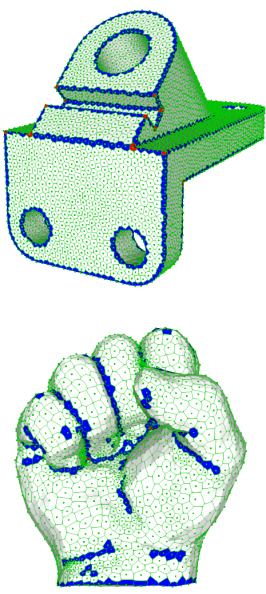

$\zeta=0.5$
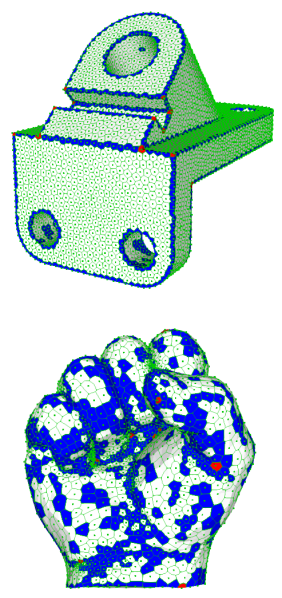

$\zeta=0.7$.

Fig. 10. Influence of the classification tolerance $\zeta$. The Voronoi cells of vertices color code the classification with red=feature vertex, blue $=$ crease vertex and white $=$ smooth vertex. We show the Anchor model with obvious features (top) and the Hand model without obvious features (bottom).

Nonlinear Hausdorff Distance Minimization: After one of the former initializations is done, we further optimize the position of a vertex $v$ by directly minimizing the

approximate two-sided Hausdorff distance of Sec. 4.2. This optimization is highly nonlinear, since changing the position of $v$ changes the samples $S_{R}$ of the modified mesh $M_{R}$ as well as the links from the input mesh samples $S_{I}$ to $M_{R}$. We perform an optimization similar to the Hausdorff distance minimization technique proposed by Winkler et al. [44]. However, we improve the feature-sensitivity by adjusting the weighting scheme with our feature intensity function $\mathcal{F}$, as detailed next.

We want to relocate $v$ in order to minimize the twosided Hausdorff distance between the local area $L \subset M_{R}$ consisting of all one-ring triangles of $v$ and the subregion of $M_{I}$ with links into $L$, referred to as $M_{I \rightarrow L}$. Assume that the corresponding subsets of samples for our Hausdorff distance approximation are $S_{L} \subset S_{R}$ and $S_{I \rightarrow L} \subset S_{I}$. Then according to Motzkin and Walsh's theorem [45] there exist weights $w_{i}$ and $\hat{w}_{i}$ such that minimizing Eq. (5) w.r.t. the center vertex $v$ is equivalent to minimizing

$$
\sum_{a_{i} \in S_{L}} w_{i}\left|a_{i}(v)-\hat{a}_{i}\right|^{2}+\sum_{b_{i} \in S_{I \rightarrow L}} \hat{w}_{i}\left|\hat{b}_{i}(v)-b_{i}\right|^{2}
$$

where $\hat{a}_{i} \in M_{I \rightarrow L}$ and $\hat{b}_{i}(v) \in L$ are the closest points to $a_{i}$ and $b_{i}$ respectively. Freezing the closest point pairs and assuming a linear barycentric relation

$$
a_{i}(v)-\hat{a}=\alpha_{i} v+\beta_{i} d_{i}+\gamma_{i} e_{i}-\hat{a}=\alpha_{i} v-p_{i}
$$

with constant $p_{i}=\hat{a}-\beta_{i} d_{i}-\gamma_{i} e_{i}$ and similarly expressed $\hat{b}_{i}(v)-b_{i}=\hat{\alpha}_{i} v-\hat{p}_{i}$, the optimal position $v^{*}$ can be computed analytically

$$
v^{*}=\frac{\sum_{a_{i}} w_{i} \alpha_{i} p_{i}+\sum_{b_{i}} \hat{w}_{i} \hat{\alpha}_{i} \hat{p}_{i}}{\sum_{a_{i}} w_{i} \alpha_{i}^{2}+\sum_{b_{i}} \hat{w}_{i} \hat{\alpha}_{i}^{2}}
$$

To find optimal weights $w_{i}$ and $\hat{w}_{i}$, Winkler et al. [44] use Lawson's algorithm [46] with iterative updates of the form

$$
w_{i}^{(k+1)}=w_{i}^{(k)} \cdot d\left(a_{i}^{(k)}, \hat{a}_{i}^{(k)}\right),
$$

where $d\left(a_{i}^{(k)}, \hat{a}_{i}^{(k)}\right)$ is the Euclidean distance of the closestpoint pair $\left(a_{i}, \hat{a}_{i}\right)$ after the $k$-th iteration, and initialization $w_{i}^{(0)}=1$. The idea behind this scheme is that samples with larger distances get a higher weight in the next iteration. Based on our feature intensity function and a sample density estimation, the weight update can be improved to

$$
w_{i}^{(k+1)}=w_{i}^{(k)} \cdot d\left(a_{i}^{(k)}, \hat{a}_{i}^{(k)}\right) \cdot \mathcal{V}\left(a_{i}^{(k)}\right) \cdot \mathcal{F}\left(a_{i}^{(k)}\right)
$$

where $\mathcal{V}\left(a_{i}\right)$ is the Voronoi cell area of sample $a_{i}$, and $\mathcal{F}\left(a_{i}\right)$ is the linearly interpolated feature intensity value of $a_{i}$. Fig. 11 illustrates the additional weighting factors. Advantages are a better feature preservation, improved robustness w.r.t. non-uniform sampling and faster convergence.

Each time when a local operator is applied, we iteratively optimize the new position of the effected vertex by minimizing Eq. (9) using the new weighting defined in Eq. (12). The optimization procedure is similar to the Expectationmaximization (EM) algorithm: in each iteration, we first calculate the optimal position $v^{*}$ of vertex $v$ using Eq. (10), and then move $v$ to $v+\lambda\left(v^{*}-v\right), \lambda \in(0,1]$ with default $\lambda=0.9$ and update the closest point pairs. In practice, a few iterations usually suffice to get very close to the optimum. 


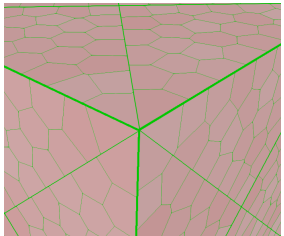

$\mathcal{V}\left(a_{i}\right)$

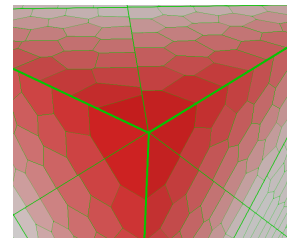

$\mathcal{F}\left(a_{i}\right)$

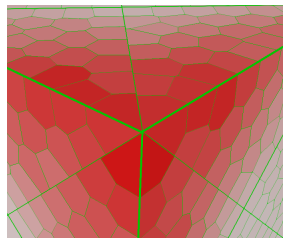

$\mathcal{V}\left(a_{i}\right) \cdot \mathcal{F}\left(a_{i}\right)$
Fig. 11. Sample weights of a cube corner, with the integration of $\mathcal{V}\left(a_{i}\right)$ and $\mathcal{F}\left(a_{i}\right)$. Higher intensity of red highlights larger weight values.

\section{Experiment Results ANd Discussions}

We implemented our approach in $\mathrm{C}++$ and tested on a 64bit Windows 8.1 operating system. The CGAL library [47] provided most of the basic data structures and operations. Timings for all the examples were conducted on a single $3.40 \mathrm{GHz}$ Intel(R) Core(TM) i7-2600K CPU with a 16GB RAM. We provide next a complete evaluation of our algorithm and comparisons with state-of-the-art approaches.

\subsection{Evaluation of the Local Error Update Scheme}

For efficient local error update we use the axis aligned bounding box tree of CGAL. As global error update is too compute-intensive we only verify how the inner patch sizes and sampling densities affect the effectiveness and efficiency of the local error update scheme (Fig. 12). We find that even when the inner patch size is set to one-ring (cf. Fig. 3), our local error update approach catches more than $99.9 \%$ of the global nearest points. The accuracy increases little with higher sampling density and larger inner patch size. We set the inner patch sizes as one-ring facets and the average sampling number per facet as ten in all experiments.
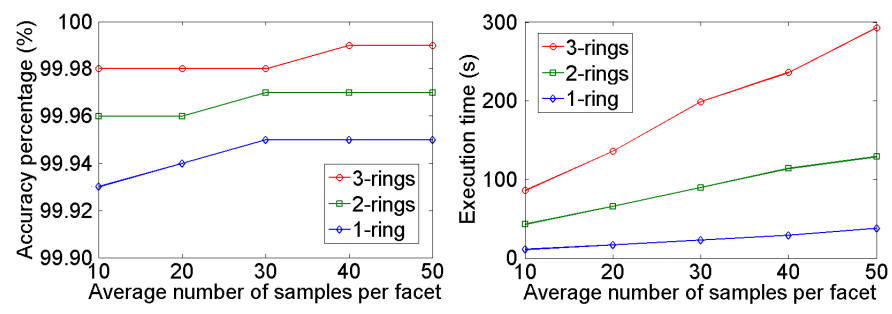

Fig. 12. The nearest point searching accuracy and execution time of our local error scheme, with respect to different sampling densities and inner patch sizes. In this experiment, $\delta=0.2 \%$ of the bounding box's diagonal length $(\% b b)$ and $\theta=30^{\circ}$. The above data are the averages of 10 consecutive executions with the Rockerarm model (Table 2) as input.

\subsection{Evaluation of Vertex Position Optimization}

Solely applying the vertex position initialization makes most vertices of $M_{R}$ stay near or on the surface of $M_{I}$. Though this quasi-interpolation preserves sharp features, the optimal geometric fidelity is usually achieved when $M_{R}$ is an approximation of $M_{I}$. We visually demonstrate the difference between interpolation and approximation in Fig. 13. In the interpolation case, the Hausdorff distance (Hdist) [48] between the sphere and the icosahedron is $6.48(\% b b)$, and the root mean square (RMS) distance is $4.84(\% b b)$; while in the approximation case, the Hdist and RMS distances between them are $5.00(\% b b)$ and $1.09(\% b b)$, respectively.

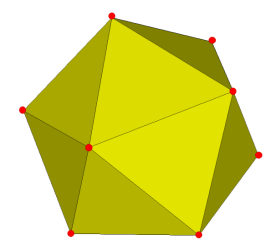

(a) Icosahedron

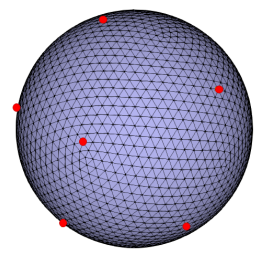

(b) Interpolation

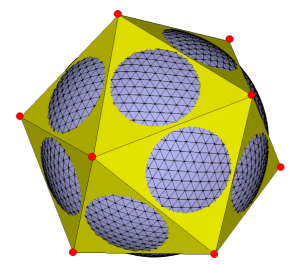

(c) Approximation
Fig. 13. Demonstration of interpolation and approximation. We use an icosahedron (a) to interpolate and approximate a sphere ((b) and (c)). In interpolation, the vertices of $M_{R}$ are guaranteed to be on the surface of $M_{R}$, while in approximation, the error is minimized, regardless of whether the vertices of $M_{R}$ are on the surface of $M_{I}$.

However, the approximation might destroy features when minimizing the local sample pair distances [44]. Fig. 14 compares the average distance and RMS distance based on Lawson's weighting scheme (Eq. (11)) and our improved weighting scheme (Eq. (12)). Generally, our weighting scheme reduces the average distance and RMS distance about $2.3 \%$ and $3.1 \%$ respectively. However, it reduces the approximation error of vertices on sharp features about $11.8 \%$ and $12.8 \%$, respectively. Therefore, applying the improved weighting scheme does not only reduce the approximation error, but also better preserves sharp features.
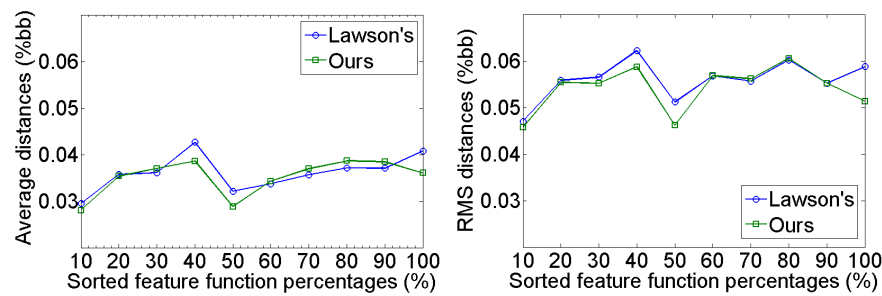

Fig. 14. Comparison of geometric fidelity between Lawson's weighing scheme [44] and ours(Eq. 12). We sort the vertices in $M_{I}$ according to their saliency function values in ascending order, and compute their average distance and RMS distance in each bin. In this experiment, $\delta=0.2(\% b b)$, and $\theta=30^{\circ}$. The above data are the averages of 10 consecutive executions with the Hand model (Fig. 10) as input.

We extensively tested how the iteration count and relocate ratio $(\lambda)$ affect the approximation error and execution time in the vertex position optimization procedure (in Fig. 15). We found that setting the iteration count to two and the relocate ratio to 0.9 achieved the best compromise between effectiveness and efficiency. This configuration is used in all our later experiments.

\subsection{Evaluation of Initial Mesh Simplification}

In general, applying Alg. 2 further reduces $20 \%$ vertices on average. However, the execution time would be 2-3 times slower than not applying it. Fig. 16 shows the remeshing results of the Egea model with and without applying initial mesh simplification, and Tab. 2 further compares the differences (OUR vs. OUR*). Usually, if users care more about the execution time than the mesh complexity, Alg. 2 can be disabled. However, we enable Alg. 2 by default for a better compromise among mesh complexity, element quality and approximation error. 

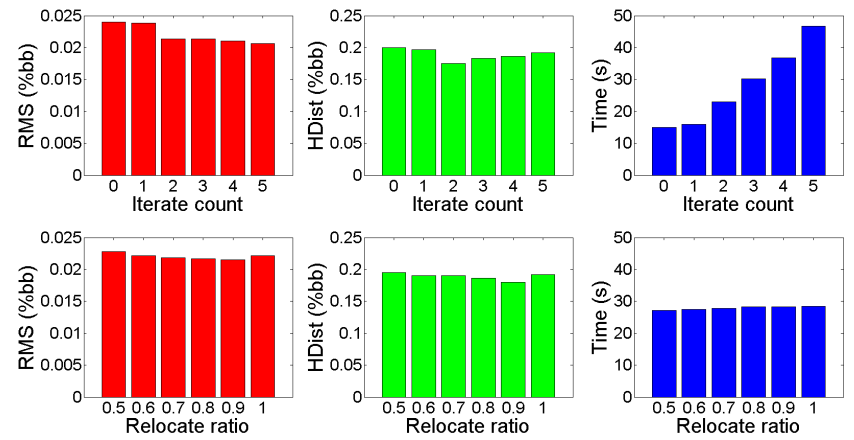

Fig. 15. Effectiveness of the iteration count and relocate ratio. For separability, RMS and Hdist bars are plotted in different scales. We set $\delta=0.2(\% b b)$, and $\theta=30^{\circ}$. The above data are the averages of 10 consecutive executions with the Homer model (Table. 2) as input.

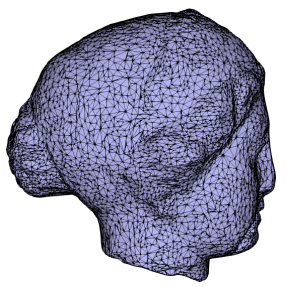

(a)

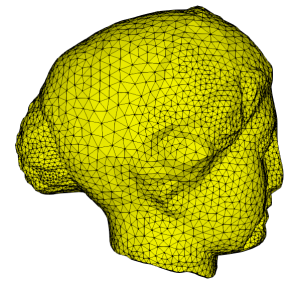

(b)

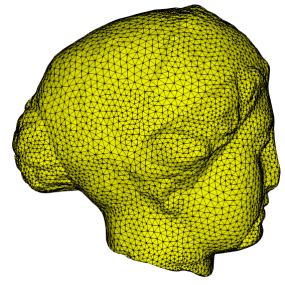

(c)
Fig. 16. Egea models with/without executing Alg. 2. we set $\theta=40^{\circ}$ and $\delta=0.20(\% b b)$. The input (a) has 8.3k vertices. The result with Alg. 2 enabled has $4.2 \mathrm{k}$ vertices (b), and spends 330 seconds; the result with Alg. 2 disabled has $7.6 \mathrm{k}$ vertices (c), and spends 133 seconds.

\subsection{Evaluation of Final Vertex Relocation}

To measure how the overall element quality is improved by applying Alg. 4, we introduce two new measurements: the first is the average minimal angles of all triangles in $M_{R}$ and the second is the average value of triangle qualities defined as $Q_{t}=2 \sqrt{3} S_{t} /\left(p_{t} h_{t}\right)$ [49], where $S_{t}$ is the area of triangle $t$, $p_{t}$ the in-radius of $t$ and $h_{t}$ the length of the longest edge in $t$. We tested the statistic element quality and the execution time with varying $\Delta \theta$ in Alg. 4 (Fig. 17), and found when $\Delta \theta<0.1^{\circ}$, the quality improvement is not significant. In our experiments, we set the default $\Delta \theta$ as $0.1^{\circ}$ in Alg. 4 .
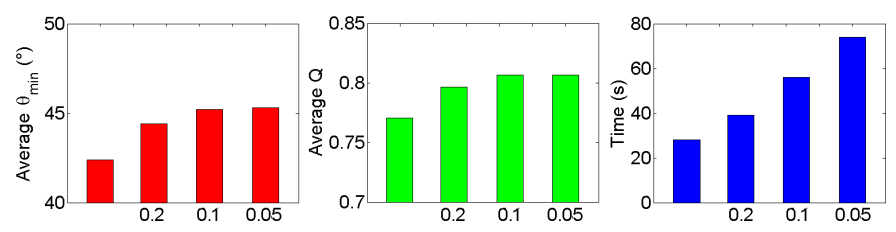

Fig. 17. The effectiveness of $\Delta \theta$ in Alg. 4. In each sub figure, the first bar indicates the value when no final vertex relocation is applied. In this experiment, $\delta=0.2(\% b b)$ and $\theta=30^{\circ}$. The above data are the averages of 10 consecutive executions with the Helmet model (Table. 2) as input.

\subsection{Influence of the Minimal Angle Threshold and the Mesh Complexity}

Our algorithm produces results with either desired minimal angle threshold or desired mesh complexity, depending on the user specified parameters. In order to get the desired minimal angle, $N$ in Alg. 1 should be set very large; otherwise, $\theta$ should be set very large (e.g. $\left.60^{\circ}\right)$. Usually, the larger the value $\theta$ or $N$ is specified, the better element quality is achieved. We tested the remeshing results of the Fandisk model with $\theta$ varying from $0^{\circ}$ to $40^{\circ}$ and $N$ varying from $0.15 \mathrm{k}$ to $2.8 \mathrm{k}$, and show the results in Fig. 1 . The complete attributes are listed in Tab. 1. Note that in Fig. 1(g), one vertex has been relocated a little away from the crease after refinement, such that the minimal angle is improved. This happens when optimizing a mesh with sharp features up to a high minimal angle threshold.

\section{TABLE 1}

Influence of $\theta$ and $\sigma . V_{567}$ is the percent of vertices with valences 5,6 and 7. We set $\delta=0.2(\% b b)$, and the Fandisk (Fig. 1(a)) is the input.

\begin{tabular}{|c|c|c|c|c|c|c|}
\hline $\boldsymbol{\theta}$ & $\# \mathbf{V}$ & $\boldsymbol{Q}_{\min }$ & $\boldsymbol{\theta}_{\boldsymbol{m a x}}$ & $\mathbf{R M S}(\mathbf{\% b b})$ & $\boldsymbol{V}_{\mathbf{5 6 7}}(\%)$ & Time \\
\hline 0 & $0.15 k$ & 0.040 & 174.4 & 0.043 & 76.5 & $1: 42^{*}$ \\
10 & $0.20 k$ & 0.188 & 155.1 & 0.038 & 71.3 & $2: 18$ \\
20 & $0.26 k$ & 0.340 & 135.5 & 0.036 & 78.6 & $2: 41$ \\
30 & $0.40 k$ & 0.482 & 117.7 & 0.031 & 81.7 & $3: 01$ \\
35 & $0.73 k$ & 0.552 & 109.3 & 0.029 & 85.5 & $3: 44$ \\
40 & $2.8 k$ & 0.640 & 98.8 & 0.022 & 98.5 & $6: 14$ \\
\hline
\end{tabular}

* This indicates the execution time of Alg. 2.

We compare our results with the state-of-the-art methods, and find only in a small portion of results presented in [19] and [9], the minimal angles exceed $35^{\circ}$ (with highest record $38^{\circ}$ ). For most other methods, the minimal angles vary between $\left[25^{\circ}, 35^{\circ}\right]$. Contrary to the previous work, our method is able to generate results with minimal angles higher than $35^{\circ}$ in all test cases. The complete comparison with the state-of-the-art methods is shown in Sec. 6.8.

\subsection{Influence of the Approximation Error Threshold}

We demonstrate the influence of $\delta$ in Fig. 18. The results indicate that $\delta$ does not influence the mesh complexity and the execution time significantly with a fixed $\theta$ value. However, two interesting phenomena are observed: 1) when $\theta \leq 35^{\circ}$, the larger the $\theta$ value is, the lower the mesh complexity we achieve; however, when $\theta$ is set to $40^{\circ}$, both mesh complexity and execution time increase dramatically. This is because when $\theta$ is small, the edge collapse operator is preferentially applied, which increases the minimal angles while reduces the mesh complexity. However, when $\theta$ is large, more edge split operators are applied to modify local connections. 2) Within a fixed $\theta$, when $\delta$ increases, the mesh complexity decreases slightly and smoothly, since the higher $\delta$ is set, the more edge collapse operators are triggered.
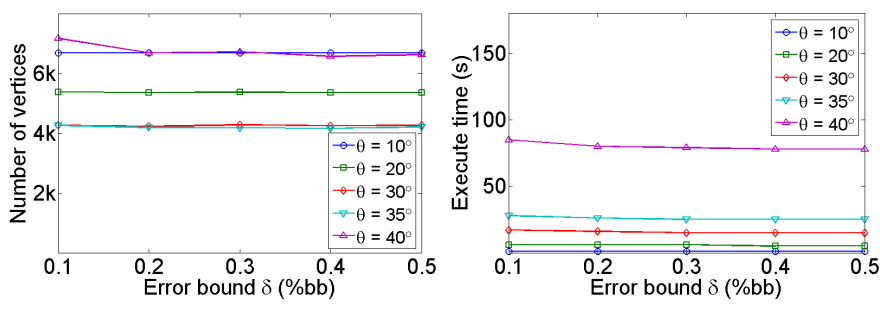

Fig. 18. Influence of the error-bound threshold $\delta$. The above data are the averages of 10 consecutive executions with the Elephant model (Table 2) as input.

We compare our results with those provided by Yan et al. [9], [19], [22]. The best record of the approximation 
error is $0.10(\% b b)$, which is generated by [8] with the Homer model as input. However, it can not be explicitly controlled. For other methods, the approximation error is between $0.3-0.5(\% b b)$, and still cannot be strictly bounded. In striking contrast, our algorithm is able to explicitly and strictly control the approximation error, and achieve the approximation error as low as $0.07(\% b b)$ with the same input. More complete comparisons are in Sec. 6.8.

\subsection{Robustness}

Since in our local error update scheme, the closest point pairs of stratified samples are reliably initialized and locally updated, our method is robust to models with complex topology, holes, and intersections of surfaces. For example, the Close spheres model (Fig. 19(c)) is composed of two rounded half spheres that are positioned very close to each other, and the Klein bottle model (Fig. 19(h)) exhibits nonorientable surfaces that are self-intersecting. Our method generates the right results for both of them.

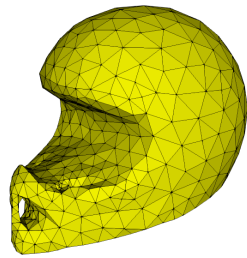

(a) Helmet

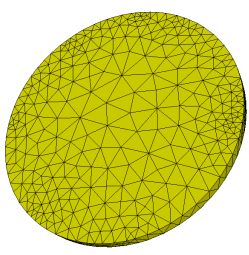

(d) Disk

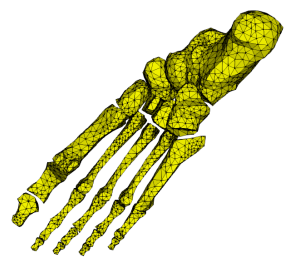

(g) Bones

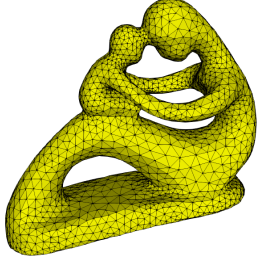

(b) Fertility

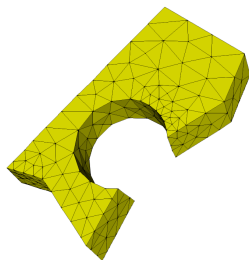

(e) U-part

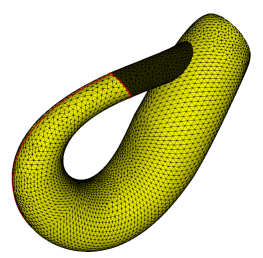

(h) Klein's bottle

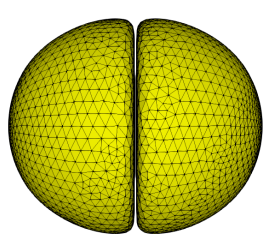

(c) Close spheres

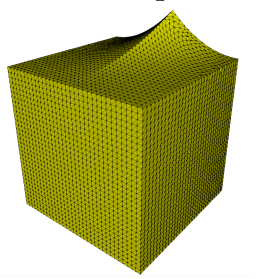

(f) Smooth crease

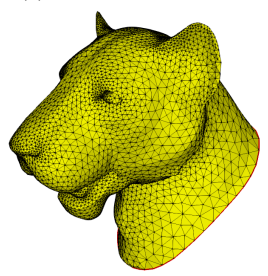

(i) Lion head
Fig. 19. Selected results. In this experiment, $\delta$ is set to $0.2 \%(b b)$ and $\theta$ is set to $35^{\circ} \cdot \ln (\mathrm{h})$ and (i), the boundaries are rendered in red. The dark part of $(h)$ means the triangle normals are inside.

By integrating the feature intensity function, our method successfully handles models with and without clear sharp features. The Helmet (Fig. 19(a)), Fertility (Fig. 19(b)) and Close spheres models are smooth, whereas the U-part (Fig. 19(e)) and the Smooth crease models (Fig. 19(f)) have sharp features. In addition, since the feature intensity also captures boundaries, our method is capable of remeshing models with boundaries (Fig. 19(i)) and smooth features (Fig. 19(f)).

Our method requires no surface parameterization, making it naturally suitable for high-genus models (Fig. 19 (a) and Fig. 19(b)) as well as models with multiple components
(Fig. 19(g)). Note that adaptivity is created automatically by the approximation error parameter $\delta$ if reasonable without requiring an a priori estimation of a density function. The Helmet, Fertility, U-part and Lion head models in Fig. 19 clearly illustrate this advantage.

Our method is suitable to process models with very high/low resolutions and/or badly shaped triangles. To tackle dense models, typically the initial mesh simplification strongly reduces the mesh complexity. For very coarse models, users can optionally increase the sampling density for better error control. We present two typical examples: the input Fertility model has $13 \mathrm{k}$ vertices, whereas our remeshing result (Fig. 19(b)) has only 2.9k vertices, thanks to the effectiveness of Alg. 2. The input U-part model possesses only 86 vertices. By sampling 50 points in each facet averagely, we get the result with 347 vertices, and the geometric fidelity is well-controlled.

\subsection{Comparisons}

We compare our approach to the state-of-the-art techniques in terms of efficiency, geometric fidelity, element quality and mesh complexity. For simplicity, only the most efficient methods (RAR [26] and MMGS, an improvement of YAMS [24]) and the methods that produce the best results in Yan and Wonka's conduction [9] (CVT [8] (100 iterations in our experiments), CVD [4] and MPS [9]) are compared with identical inputs. Among all the compared methods, CVT and CVD require the number of vertices to be specified, and the Hdist is required in RAR and MMGS. To make the results comparable, we set the Hdist of RAR to the same value as that of our method, and carefully adjusted the Hdist parameter for MMGS, such that it generates results with the same complexity as CVT, MPS and CVD. In our method, OUR* means Alg. 2 is disabled while OUR means it is enabled. A detailed comparison is listed in Tab. 2, and Fig. 20 shows a close-up comparison. More visual comparisons are provided in the supplemental materials, available online.

From all compared methods, RAR performs most efficiently and introduces the lowest mesh complexity. However, the geometric fidelity cannot be guaranteed. MMGS is also efficient, yet it introduces much higher Hdist distances. Our method is almost at the same level of efficiency as CVT when $\theta=35^{\circ}$, but is much slower when $\theta=40^{\circ}$.

According to [9], CVT performs best in keeping high geometric fidelity, but cannot explicitly bound the approximation error. By setting $\delta$ comparable to CVT's best results, our method consistently produces results with strictly bounded Hdist, and produces lower Hdist and RMS distances in most cases. We also find that our method consistently generates results with higher minimal angle and triangle quality than MPS, due to the fact that our method provides a better improvement of "worst element quality" measured by the minimal angle. However, since our method does not optimize the global connectivity of the input, our results have lower regularity (measured as $V_{567}$ ) than MPS.

From Tab. 2, we see that by setting the desired resolution lower than MMGS, CVT, MPS and CVD, we still get results with higher geometric fidelity and better bounds of minimal angle. For the very dense Bunny model, when Alg. 2 is enabled, our method even reduces the complexity of the 
TABLE 2

Comparison with the state-of-the-art methods. For all the method, the input parameters are highlighted with italic fonts, and the best results are highlighted in bold. In column Hdist (\%bb), the values before "/" are the input, and the values after "/" are the real HDist measured by Metro [48].

\begin{tabular}{|c|c|c|c|c|c|c|c|c|c|c|}
\hline Input & Methods & $\# \boldsymbol{V}$ & $Q_{\min }$ & $\theta_{\min }\left(^{\circ}\right)$ & $\theta_{\max }\left(^{\circ}\right)$ & Hdist $(\% \mathrm{bb})$ & RMS (\%bb) & $\boldsymbol{\theta}<\mathbf{3 0}^{\circ}(\%)$ & $V_{567}(\%)$ & Time \\
\hline \multirow{8}{*}{$\begin{array}{c}\text { Rockerarm } \\
(3.4 \mathrm{k})\end{array}$} & [RAR] & $2.1 \mathrm{k}$ & 0.556 & 27.9 & 107.2 & $0.20 / 0.94$ & 0.120 & 0.02 & 100 & $<0: 01$ \\
\hline & [MMGS] & $5.8 \mathrm{k}$ & 0.056 & 3.4 & 172.5 & 0.47 & 0.103 & 1.73 & 93.4 & $0: 01$ \\
\hline & [CVT] & $5.8 k$ & 0.588 & 28.3 & 104.6 & 0.21 & 0.030 & 0.02 & 99.9 & $0: 48$ \\
\hline & [MPS] & $5.8 k$ & 0.516 & 32.0 & 113.6 & 0.48 & 0.033 & 0 & 100 & $0: 05$ \\
\hline & [OUR*] & $2.8 \mathrm{k}$ & 0.559 & 35.0 & 108.5 & $0.20 / 0.20$ & 0.025 & 0 & 89.0 & $0: 29$ \\
\hline & {$\left[\mathrm{OUR}^{*}\right]$} & $3.0 k$ & 0.612 & 38.6 & 102.2 & $0.20 / 0.17$ & 0.024 & 0 & 95.4 & $0: 38$ \\
\hline & {$\left[\mathrm{OUR}^{*}\right]$} & $3.8 \mathrm{k}$ & 0.646 & 40.0 & 98.1 & $0.20 / \mathbf{0 . 1 4}$ & 0.020 & $\mathbf{0}$ & 98.5 & $0: 51$ \\
\hline & [OUR] & $3.6 \mathrm{k}$ & 0.639 & 40.0 & 99.0 & $0.20 / 0.20$ & 0.025 & 0 & 97.9 & $2: 58$ \\
\hline \multirow{8}{*}{$\begin{array}{c}\text { Homer } \\
(6.0 \mathrm{k})\end{array}$} & [RAR] & $2.6 \mathrm{k}$ & 0.569 & 29.2 & 106.5 & $0.20 / 0.55$ & 0.070 & 0.02 & 99.9 & $<0: 01$ \\
\hline & [MMGS] & $7.2 \mathrm{k}$ & 0.210 & 13.1 & 152.2 & 0.43 & 0.028 & 1.07 & 95.8 & $0: 01$ \\
\hline & [CVT] & $7.2 k$ & 0.568 & 25.3 & 102.3 & 0.10 & 0.021 & 0.02 & 99.9 & $1: 14$ \\
\hline & [MPS] & $7.2 k$ & 0.513 & 32.0 & 115.0 & 0.31 & 0.023 & 0 & 100 & $0: 05$ \\
\hline & [OUR* ${ }^{*}$ & $4.8 \mathrm{k}$ & 0.553 & 35.0 & 109.2 & $0.20 / 0.09$ & 0.010 & 0 & 91.8 & $0: 46$ \\
\hline & {$\left[\mathrm{OUR}^{*}\right]$} & $5.0 k$ & 0.600 & 37.8 & 103.6 & $0.20 / 0.09$ & 0.010 & 0 & 95.2 & $0: 59$ \\
\hline & [OUR*] & $6.9 \mathrm{k}$ & 0.643 & 40.0 & 98.5 & $0.20 / 0.07$ & 0.009 & 0 & 98.7 & $1: 29$ \\
\hline & [OUR] & $4.3 \mathrm{k}$ & 0.635 & 40.0 & 99.5 & $0.20 / 0.17$ & 0.018 & 0 & 97.8 & $4: 48$ \\
\hline \multirow{8}{*}{$\begin{array}{c}\text { Triceratops } \\
(2.8 \mathrm{k})\end{array}$} & [RAR] & $1.6 \mathrm{k}$ & 0.607 & 30.0 & 98.4 & $0.20 / 2.61$ & 0.570 & 0.03 & 99.8 & $<0: 01$ \\
\hline & [MMGS] & $9.0 \mathrm{k}$ & 0.270 & 13.5 & 143.3 & 0.41 & 0.080 & 1.11 & 93.6 & $0: 01$ \\
\hline & [CVT] & $9.0 k$ & 0.543 & 31.7 & 110.3 & 0.12 & 0.018 & 0 & 99.9 & $1: 23$ \\
\hline & [MPS] & $9.0 k$ & 0.506 & 32.0 & 114.8 & 0.46 & 0.062 & 0 & 100 & $0: 29$ \\
\hline & [OUR*] & $2.1 \mathrm{k}$ & 0.552 & 35.0 & 109.3 & $0.20 / 0.16$ & 0.028 & 0 & 87.0 & $0: 28$ \\
\hline & [OUR*] & $3.0 k$ & 0.605 & 38.4 & 103.0 & $0.20 / 0.18$ & 0.024 & 0 & 93.8 & $0: 41$ \\
\hline & [OUR* $\left.{ }^{*}\right]$ & $4.8 \mathrm{k}$ & 0.634 & 40.0 & 99.6 & $0.20 / 0.19$ & 0.036 & 0 & 97.7 & $1: 39$ \\
\hline & [OUR] & $3.5 \mathrm{k}$ & 0.644 & 40.0 & 98.4 & $0.20 / 0.19$ & 0.040 & 0 & 97.1 & $2: 36$ \\
\hline \multirow{8}{*}{$\begin{array}{c}\text { Elephant } \\
(6.9 \mathrm{k})\end{array}$} & [RAR] & $2.9 \mathrm{k}$ & 0.480 & 24.9 & 115.5 & $0.20 / 5.0$ & 0.112 & 0.12 & 100 & $<0: 01$ \\
\hline & [MMGS] & $11 \mathrm{k}$ & 0.187 & 10.9 & 155.1 & 0.29 & 0.034 & 1.24 & 93.5 & $0: 02$ \\
\hline & [CVT] & $11 k$ & 0.560 & 26.8 & 107.7 & 0.11 & 0.018 & 0.01 & 99.8 & $1: 43$ \\
\hline & [MPS] & $11 k$ & 0.505 & 32.0 & 114.9 & 0.38 & 0.061 & 0 & 100 & $0: 31$ \\
\hline & [OUR*] & $4.4 \mathrm{k}$ & 0.553 & 35.0 & 109.2 & $0.20 / \mathbf{0 . 1 1}$ & 0.014 & 0 & 90.4 & $0: 49$ \\
\hline & {$\left[\mathrm{OUR}^{*}\right]$} & $5.0 k$ & 0.617 & 39.1 & 101.5 & $0.20 / 0.13$ & 0.013 & 0 & 96.6 & $1: 05$ \\
\hline & [OUR*] & $6.8 \mathrm{k}$ & 0.638 & 40.0 & 99.1 & $0.20 / \mathbf{0 . 1 1}$ & 0.010 & 0 & 98.7 & $1: 45$ \\
\hline & [OUR] & $2.7 \mathrm{k}$ & 0.633 & 40.0 & 99.7 & $0.20 / 0.14$ & 0.021 & 0 & 98.4 & $4: 25$ \\
\hline \multirow{8}{*}{$\begin{array}{c}\text { Bunny } \\
(34 \mathrm{k})\end{array}$} & [RAR] & $4.1 \mathrm{k}$ & 0.545 & 27.5 & 109.4 & $0.20 / 0.69$ & 0.072 & 0.02 & 100 & $<0: 01$ \\
\hline & [MMGS] & $12 \mathrm{k}$ & 0.260 & 13.1 & 142.4 & 0.41 & 0.041 & 0.59 & 93.8 & $0: 03$ \\
\hline & [CVD] & $12 k$ & 0.150 & 9.6 & 160.1 & 0.34 & 0.028 & 0.71 & 96 & $0: 05$ \\
\hline & {$[\mathrm{CVT}]$} & $12 k$ & 0.603 & 30.6 & 103.1 & 0.20 & 0.018 & 0 & 99.9 & $3: 57$ \\
\hline & [MPS] & $12 k$ & 0.510 & 32.0 & 114.6 & 0.37 & 0.035 & 0 & 100 & $0: 24$ \\
\hline & [OUR*] & $37 \mathrm{k}$ & 0.550 & 35.0 & 109.6 & $0.20 / \mathbf{0 . 0 7}$ & 0.003 & 0 & 95.8 & $3: 23$ \\
\hline & [OUR] & $1.5 k$ & 0.637 & 39.8 & 99.2 & $0.20 / 0.19$ & 0.033 & 0 & 97.2 & $10: 41$ \\
\hline & [OUR] & $1.7 \mathrm{k}$ & 0.646 & 40.0 & 98.2 & $0.20 / 0.19$ & 0.031 & 0 & 97.7 & $12: 28$ \\
\hline
\end{tabular}

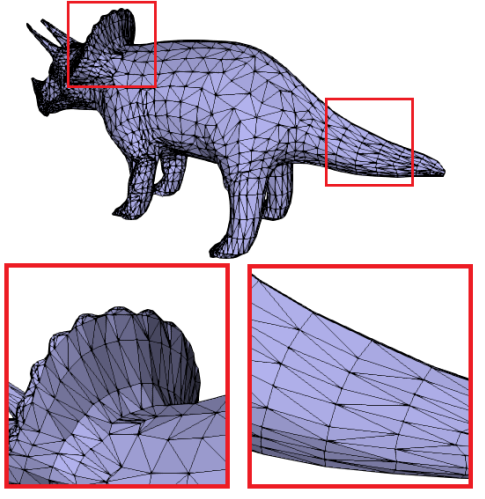

(a) Input Triceratops model
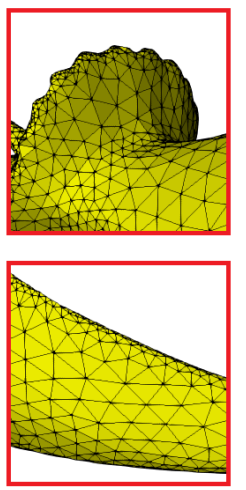

(b) RAR
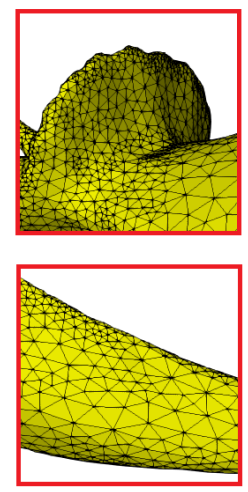

(c) MMGS

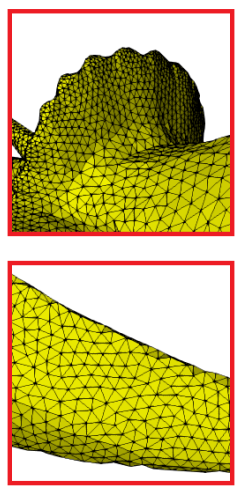

(d) CVT
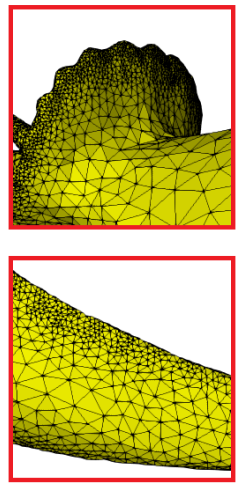

(e) MPS
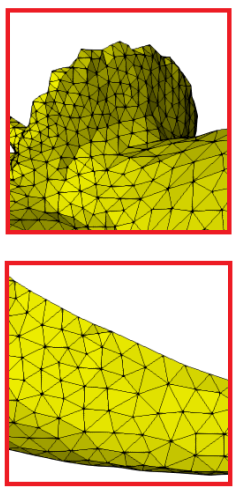

(f) OUR

Fig. 20. A close-up comparison of results with state-of-the-art approaches. In our method, $\delta$ is set to $0.20(\% b b)$ and $\theta$ is set to $40^{\circ}$.

Bunny model to $5 \%$ of the input without violating the errorbound constraint. When it is disabled, the resolutions are still lower than the inputs in most cases, since the edge collapse operator has high priority in Alg. 1.

To the best of our knowledge, RAR, MMGS, CVT and MPS require sharp features to be specified or detected in advance, which may be time-consuming or error-prone. Though CVD is able to preserve features implicitly, it leads to results with lower geometric fidelity and element quality than our method.

Since our method does not optimize the element quality globally, the average element quality is not superior to 
remeshing based on farthest point optimization [22] (FPO). However, we consistently produce results with better geometric fidelity and larger minimal angle than FPO.

\subsection{Limitations}

Although practically the minimal angles can be improved to values above $35^{\circ}$ in all our test cases, we do not have any theoretical guarantee for the convergence with a specified $\theta$. To challenge our algorithm, we set $\theta$ to its theoretical upper bound and show the best results that our algorithm achieved in Tab. 3. We found the approximation error can still be bounded. However, the algorithm runs into infinite loops or generates degenerated edges while refining.

Another limitation is that we can only tackle 2-manifold meshes, for the reason that our local operators highly rely on the topology information of local regions. Finally, our method cannot remesh noisy models robustly, since the feature intensity function will interpret the noise as some kind of features and thus tries to preserve it.

TABLE 3

Cases when too high $\theta$ is specified. We set $\theta=60^{\circ}$, run Alg. 1 until it fails, and record the best results it achieved. Alg. 2 is enabled here.

\begin{tabular}{|c|c|c|c|c|}
\hline Input & \#V & $\boldsymbol{\theta}_{\min }$ & Hdist(\%bb) & Fail Types \\
\hline Rockerarm & $4.2 \mathrm{k}$ & 41.22 & $0.20 / 0.19$ & Degenerated edges \\
Homer & $6.1 \mathrm{k}$ & 41.13 & $0.20 / 0.14$ & Infinite loops \\
Triceratops & $4.8 \mathrm{k}$ & 40.02 & $0.20 / 0.19$ & Degenerated edges \\
Elephant & $4.8 \mathrm{k}$ & 41.23 & $0.20 / 0.13$ & Infinite loops \\
Bunny & $2.4 \mathrm{k}$ & 41.42 & $0.20 / 0.19$ & Infinite loops \\
\hline
\end{tabular}

\section{Conclusion}

We presented a novel surface remeshing algorithm based on minimal angle improvement. In this framework, the minimal angle of the input model is sequentially improved by applying local operators. Furthermore, an efficient and reliable local error update scheme was designed and embedded for explicitly bounding the approximation error, and two novel feature intensity functions were defined and integrated in the vertex relocation, in order to preserve features implicitly. Compared to the state-of-the-art, our method consistently generates results with higher element quality and lower mesh complexity but satisfied error-bounds. The resulting meshes are well suited for robust numerical simulations, since they offer bounded approximation error and large minimal angles.

However, there are still some limitations (Sec. 6.9), which motivate future work in the following aspects: 1) providing a theoretical convergence guarantee with a specified $\theta ; 2)$ extending the current implementation such that this framework can be applied to triangle soups or even point clouds, for error-bounded and feature preserving mesh reconstruction; and 3) exploring more robust feature intensity functions for measuring sharp features on noisy models.

\section{ACKNOWLEDGMENTS}

We wish to thank Mario Botsch for providing the source code and results of their method, and Pascal Frey for providing the MMGS software. This work was partially supported by the European Research Council (ERC Starting Grant Robust Geometry Processing \#257474), the National Science Foundation of China $(61373071,61372168,61620106003)$ and the German Research Foundation (DFG, grant GSC 111, Aachen Institute for Advanced Study in Computational Engineering Science).

\section{REFERENCES}

[1] M. Botsch, L. Kobbelt, M. Pauly, P. Alliez, and B. Lévy, Polygon Mesh Processing. AK Peters, 2010.

[2] J. R. Shewchuk, "What is a good linear element? interpolation, conditioning, and quality measures," in IMR, 2002, pp. 115-126.

[3] P. Alliez, É. C. de Verdière, O. Devillers, and M. Isenburg, "Centroidal Voronoi diagrams for isotropic surface remeshing," Graphical Models, vol. 67, no. 3, pp. 204-231, 2005.

[4] S. Valette, J.-M. Chassery, and R. Prost, "Generic remeshing of 3D triangular meshes with metric-dependent discrete Voronoi diagrams," IEEE Trans. Vis. Comput. Graph., vol. 14, no. 2, pp. 369 381, 2008.

[5] D. Cohen-Steiner, P. Alliez, and M. Desbrun, "Variational shape approximation," ACM Trans. Graph., vol. 23, no. 3, pp. 905-914, 2004.

[6] M. Mandad, D. Cohen-Steiner, and P. Alliez, "Isotopic approximation within a tolerance volume," ACM Trans. Graph., vol. 34, no. 4 pp. 64:1-64:12, 2015.

[7] P. Alliez, M. Meyer, and M. Desbrun, "Interactive geometry remeshing," ACM Trans. Graph., vol. 21, no. 3, pp. 347-354, 2002.

[8] D.-M. Yan, B. Lévy, Y. Liu, F. Sun, and W. Wang, "Isotropic remeshing with fast and exact computation of restricted Voronoi diagram," Comput. Graph. Forum, vol. 28, no. 5, pp. 1445-1454, 2009.

[9] D.-M. Yan and P. Wonka, "Gap processing for adaptive maximal Poisson-disk sampling," ACM Trans. Graph., vol. 32, no. 5, pp. 148:1-148:15, Oct. 2013.

[10] H. Hoppe, T. DeRose, T. Duchamp, J. McDonald, and W. Stuetzle, "Mesh optimization," in SIGGRAPH, 1993, pp. 19-26.

[11] M. Botsch and L. Kobbelt, "A remeshing approach to multiresolution modeling," in Symposium on Geometry Processing, 2004, pp. 185-192.

[12] P. Alliez, G. Ucelli, C. Gotsman, and M. Attene, "Recent advances in remeshing of surfaces," in Shape Analysis and Structuring, Mathematics and Visualization. Springer, 2008, pp. 53-82.

[13] Q. Du, V. Faber, and M. Gunzburger, "Centroidal Voronoi tessellations: applications and algorithms," SIAM Review, vol. 41, pp. 637-676, 1999.

[14] P. Alliez, É. C. de Verdière, O. Devillers, and M. Isenburg, "Isotropic surface remeshing," in Shape Modeling International, 2003, pp. 49-58.

[15] V. Surazhsky, P. Alliez, and C. Gotsman, "Isotropic remeshing of surfaces: A local parameterization approach," in $I M R, 2003$, pp. 215-224.

[16] V. Surazhsky and C. Gotsman, "Explicit surface remeshing," in Symposium on Geometry Processing, ser. ACM International Conference Proceeding Series, vol. 43, 2003, pp. 20-30.

[17] S. Fuhrmann, J. Ackermann, T. Kalbe, and M. Goesele, "Direct resampling for isotropic surface remeshing," in $V M V, 2010$, pp. 9-16.

[18] J. Vorsatz, C. Rössl, and H.-P. Seidel, "Dynamic remeshing and applications," J. Comput. Inf. Sci. Eng., vol. 3, no. 4, pp. 338-344, 2003.

[19] D.-M. Yan, G. Bao, X. Zhang, and P. Wonka, "Low-resolution remeshing using the localized restricted Voronoi diagram," IEEE Trans. Vis. Comput. Graph., vol. 20, no. 10, pp. 1418-1427, 2014.

[20] D.-M. Yan and P. Wonka, "Non-obtuse remeshing with centroidal Voronoi tessellation," IEEE Trans. Vis. Comput. Graph., vol. 22, no. 9, pp. 2136-2144, 2016.

[21] J. Guo, D.-M. Yan, X. Jia, and X. Zhang, "Efficient maximal Poisson-disk sampling and remeshing on surfaces," Computers $\mathcal{E}$ Graphics, vol. 46, pp. 72-79, 2015.

[22] D.-M. Yan, J. Guo, X. Jia, X. Zhang, and P. Wonka, "Blue-noise remeshing with farthest point optimization," Computer Graphics Forum, vol. 33, no. 5, pp. 167-176, 2014

[23] A. G. M. Ahmed, J. Guo, D.-M. Yan, X. Zhang, and O. Deussen, "A simple push-pull algorithm for blue-noise sampling," IEEE Trans. Vis. Comput. Graph., 2016, accepted. 
[24] P. Frey and P. George, Mesh generation, application to finite elements. Hermès Science Publ., Paris, Oxford, 814 pages, 2000.

[25] R. Narain, A. Samii, and J. F. O'Brien, "Adaptive anisotropic remeshing for cloth simulation," ACM Trans. Graph., vol. 31, no. 6, p. 152, 2012.

[26] M. Dunyach, D. Vanderhaeghe, L. Barthe, and M. Botsch, "Adaptive remeshing for real-time mesh deformation," Eurographics short papers. Eurographics Association, Girona, Spain, pp. 29-32, 2013.

[27] S. A. Lloyd, "Least squares quantization in PCM," IEEE Transactions on Information Theory, vol. 28, no. 2, pp. 129-137, 1982.

[28] M. Garland and P. S. Heckbert, "Surface simplification using quadric error metrics," in SIGGRAPH, 1997, pp. 209-216.

[29] H. Borouchaki and P. Frey, "Simplification of surface mesh using Hausdorff envelope," Computer methods in applied mechanics and engineering, vol. 194, no. 48, pp. 4864-4884, 2005.

[30] Y. Sun, D. L. Page, J. K. Paik, A. Koschan, and M. A. Abidi, "Triangle mesh-based edge detection and its application to surface segmentation and adaptive surface smoothing," in ICIP, 2002, pp. 825-828.

[31] X. Jiao and M. T. Heath, "Feature detection for surface meshes," in Proceedings of 8th international conference on numerical grid generation in computational field simulations, 2002, pp. 705-714.

[32] J. Chen, X. Ge, L.-Y. Wei, B. Wang, Y. Wang, H. Wang, Y. Fei, K.-L. Qian, J.-H. Yong, and W. Wang, "Bilateral blue noise sampling," ACM Trans. Graph., vol. 32, no. 6, pp. 216:1-216:11, 2013.

[33] Z. Zhong, X. Guo, W. Wang, B. Lévy, F. Sun, Y. Liu, and W. Mao, "Particle-based anisotropic surface meshing," ACM Trans. Graph., vol. 32, no. 4, p. 99, 2013.

[34] L. Kobbelt and M. Botsch, "Feature sensitive mesh processing," in Proceedings of the 19th Spring Conference on Computer Graphics, ser. SCCG '03, 2003, pp. 17-22.

[35] B. Lévy and Y. Liu, " $L_{p}$ centroidal Voronoi tessellation and its applications," ACM Transactions on Graphics, vol. 29, no. 4, pp. 119:1-11, 2010.

[36] J. Vorsatz, C. Rössl, L. Kobbelt, and H.-P. Seidel, “Feature sensitive remeshing," Comput. Graph. Forum, vol. 20, no. 3, pp. 393-401, 2001.

[37] W. Jakob, M. Tarini, D. Panozzo, and O. Sorkine-Hornung, "Instant field-aligned meshes," ACM Transactions on Graphics (Proceedings of SIGGRAPH ASIA), vol. 34, no. 6, Nov. 2015.

[38] M. C. Rivara, "New mathematical tools and techniques for the refinement and/or improvement of unstructured triangulations," in $I M R, 1996, \mathrm{pp} .77-86$

[39] H. Edelsbrunner, Geometry and topology for mesh generation. Cambridge University Press, 2006.

[40] J. Henrikson, "Completeness and total boundedness of the hausdorff metric," MIT Undergraduate Journal of Mathematics, vol. 1, pp. 69-80, 1999

[41] M. Tang, M. Lee, and Y. J. Kim, "Interactive Hausdorff distance computation for general polygonal models," ACM Trans. Graph., vol. 28, no. 3, 2009.

[42] J. Tournois, P. Alliez, and O. Devillers, "2D Centroidal Voronoi Tessellations with Constraints," Numerical Mathematics: Theory, Methods and Applications, vol. 3, no. 2, pp. 212-222, 2010.

[43] P. Alliez, S. Tayeb, and C. Wormser, "3D fast intersection and distance computation," in CGAL User and Reference Manual. CGAL Editorial Board, 2016.

[44] T. Winkler, K. Hormann, and C. Gotsman, "Mesh massage," The Visual Computer, vol. 24, no. 7-9, pp. 775-785, 2008.

[45] T. S. Motzkin and J. L. Walsh, "Polynomials of best approximation on a real finite point set," Trans. Am. Math. Soc., vol. 92, no. 2, pp. 231-245, 1959.

[46] C. Lawson, Contributions to the Theory of Linear Least Maximum Approximation. UCLA-Mathematics., 1961.

[47] "CGAL, Computational Geometry Algorithms Library," http://www.cgal.org.

[48] P. Cignoni, C. Rocchini, and R. Scopigno, "Metro: measuring error on simplified surfaces," in Computer Graphics Forum, vol. 17, no. 2 1998, pp. 167-174.

[49] P. J. Frey and H. Borouchaki, "Surface mesh evaluation," in IMR, 1997, pp. 363-374.

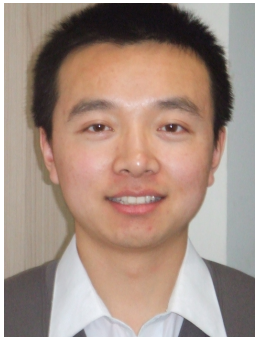

Kaimo Hu received the Bachelor and $\mathrm{PhD}$ degrees from the Tsinghua University in 2006 and 2012 , respectively. He is a post doctoral research assistant in the Department of Computer Graphics Technology Department at Purdue University. His research interests include computer graphics, shape analysis, geometric processing and procedural modeling.

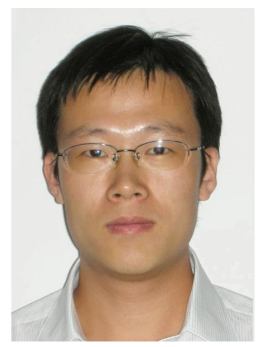

Dong-Ming Yan received the Bachelor and Master degrees from the Tsinghua University in 2002 and 2005, respectively, and the $\mathrm{PhD}$ degree from the Hong Kong University, in 2010. He is an associate professor in the National Laboratory of Pattern Recognition, Institute of Automation, Chinese Academy of Sciences. His research interests include computer graphics, geometric processing, and visualization.

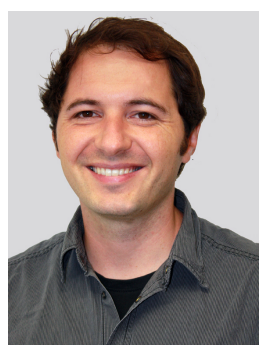

David Bommes is an assistant professor in the Computer Science department at RWTH Aachen University. In May 2016 he received the EUROGRAPHICS Young Researcher Award. He is leading the Mesh Generation and Optimization group, which scientifically contributes to various areas of geometry processing, in particular direction fields, parametrization, quad/hex mesh generation, and nonlinear/mixed-integer optimization.

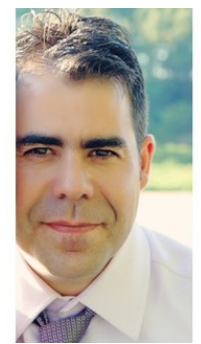

Pierre Alliez is a Senior Researcher and team leader at Inria Sophia Antipolis. He obtained his Ph.D. in Computer Science in 2000 from Telecom ParisTech. He published on topics commonly referred to as geometry processing. $\mathrm{He}$ was awarded in 2011 a Starting Grant from the European Research Council on Robust Digital Geometry Processing.

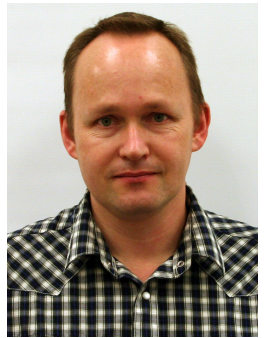

Bedrich Benes is a professor in the Computer Graphics Technology department at Purdue University. He obtained his Ph.D. and M.S. degree from the Czech Technical University. His research is primarily in the area of procedural modeling, real-time rendering, and 3D computer graphics in general. To date he has published over 100 peer reviewed publications. 